

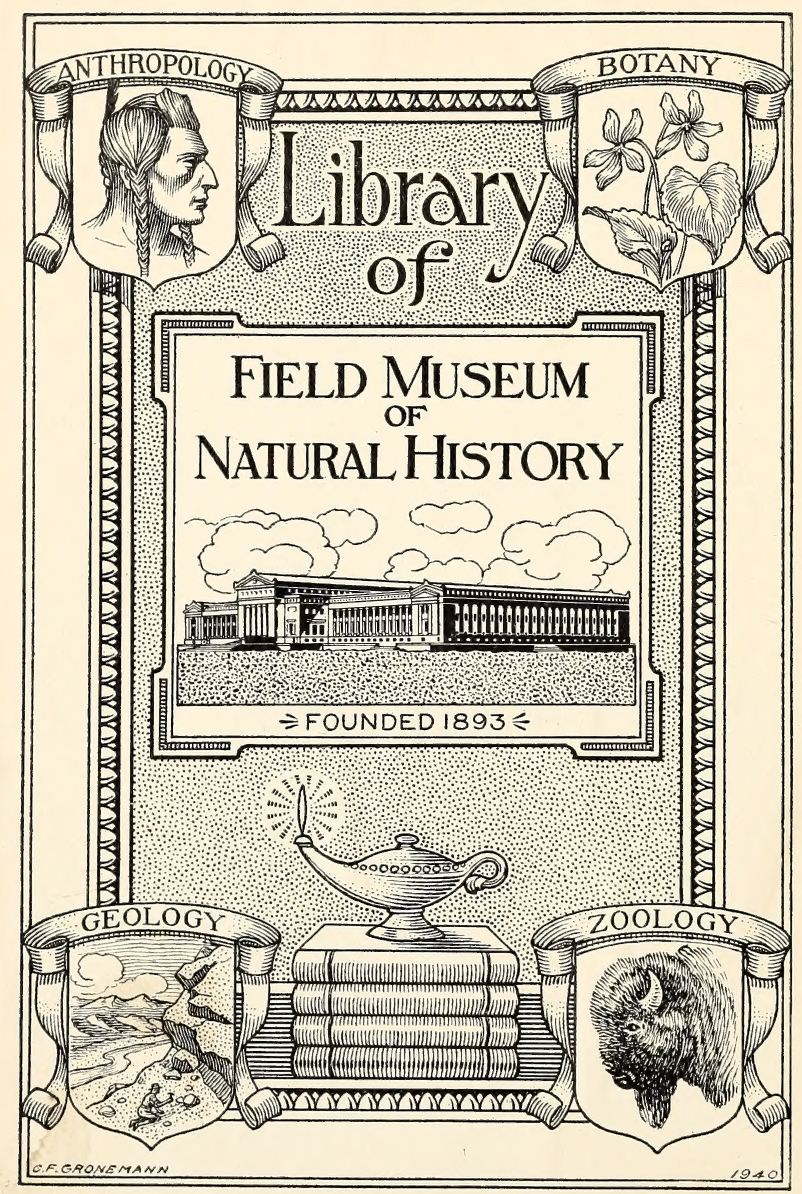



Digitized by the Internet Archive in 2011 with funding from The Field Museum's Africa Council 

MEIANIDEES IAC NYASSA

BOURGUIGNAT 



\title{
MÉLA NIDÉES
}

\author{
DU LAC NYASSA \\ SUIVIES D'UN APERÇU COMPARATIF \\ SUR 3
}

LA FAUNE MALAGOLOGIQUE DE GE LAC

AVEG GELLE DU GRAND LAG TANGANIKA

PAR

\section{J. R. BOURGUIGNAT}

SECRÉTAIRE GÉNÉRAL

Les Mélanidées du lac Nyassa, que je vais faire connaître, m'ont été remises par le célèbre voyageur Victor Giraud en mai 1885, en même temps que les nombreuses Espèces fluviales du lac Tanganika, décrites par moi en août de la même année ${ }^{1}$.

J'avais laissé de côté ces Mélanidées dans l'intention de ne les examiner qu'après mes travaux projetés sur le lac Tanganika. Actuellement que le travail d'ensemble sur la faune de ce lac est terminé $^{2}$, je passe aux Espèces du lac Nyassa. Ces Espèces, bien que peu nombreuses, sont fort intéressantes pour la connaissance de la faune mala-

1. Paris, 1885.1 vol. in-8.

2. 2 vol. in-8, avec $35 \mathrm{pl}$.

Bull. Soc. malac. France. VI. Juin 1889. - 1 
Un mot maintenant sur le lac Nyassa.

Ce lac, situé au nord antarctique et un peu à l'est du Tanganika, est formé, ainsi que celui-ci, par une longue dépression s'étendant du Sud au Nord sur une longueur de plus de 600 kilomètres. Il offre l'aspect d'une large vallée dont les sinuosités se correspondent d'un versant à l'autre; sa plus grande largeur est de 90 à 100 kilomètres, sa plus petite de 24 kilomètres. Son étendue, de 30000 kilomètres carrés, est un peu moindre que celle du Tanganika, qui atteint 39000 kilomètres, et bien inférieure à celle du Nyanza Oukéréwé, qui est plus du double ( 66500 kilom. carrés). Les côtes de ce lac sont abruptes; les falaises plongent à pic, et des sondes de 200 mètres n'ont pu en quelques endroits atteindre le fond. Sur la côte orientale règne la belle chaîne Livingstone, dont les sommets atteignent jusqu'à 2000 mètres, chaîne qui va en s'abaissant graduellement du Sud au Nord. Du côté occidental se développent de hautes collines formant saillie sur une série continue de plateaux et quelques hauts massifs isolés, parfois fort élevés, comme celui du Tchombé, qui s'élance à 1700 mètres.

Le Nyassa, dont l'altitude est de 480 mètres, n'est alimenté que par de petites rivières. Les plus importantes sont sur la rive occidentale. Ses eaux, d'une limpidité parfaite, puisqu'elles ne laissent aucune trace de sédiments dans les chaudières à vapeur et sur le doublage en cuivre des bateaux, se déversent, à l'extrémité Nord, dans une large 
rivière, le Shiré, qui, à 100 kilomètres de là, se jette dans un bras du Zambèse.

On rencontre à ce qu'il paraît fort peu d'atterrissements et de plages sablonneuses dans ce lac, à l'exception des plages basses des environs de Livingstonia, petite ville à l'extrémité septentrionale, d'où partent les bateaux, et celles de Makura à l'extrémité méridionale, de Karonga, de KotaKota, etc. Sur la rive occidentale, presque tout le reste des côtes est abrupt.

Ce lac est encore inconnu au point de vue malacologique. Je ne connais guère que MM. J. Kirk et F.-A. Simons, pasteurs anglicans, qui se soient donné la peine de recueillir quelques Espèces. Le champ de leur exploration n'a guère dépassé les rives septentrionales, à partir du Shiré jusqu'à celles qui avoisinent la petite ville de Livingstonia. Les Espèces recueillies par le voyageur Victor Giraud proviennent de l'autre extrémité du lac, aux environs de Karonga. On verra, par la bizarrerie et la singularité des formes, combien ce lac doit renfermer de richesses malacologiques et à quel point il serait précieux pour la science que ce lac soit exploré par une personne zélée, au courant des recherches zoologiques.

\section{$\S 2$.}

Les Mélanidées du lac Nyassa appartiennent aux genres Melania, Nyassia, Nyassella, Micronyassia et Nyassomelania. 


\section{MELANIA}

\section{MELANIA TUBERGULATA}

Melania tuberculata, Bourguignat, Moll. orient, p. 65,1853 , et Malac. Alg., II, p. 251, pl. xv, fig. 1-11, 1864. (Nerita tuberculata, de Müller, 1774. Melania fasciolata, de Lamarck, 1822, etc.)

Ėspèce des plus cosmopolites. Les échantillons du Nyassa sont bien semblables à ceux du Tanganika, du Zambèse, du Kyngani, près de Bayamoyo, et des autres rivières de l'Oussaghara et du Mozambique.

\section{NYASSIA}

Les Espèces comprises sous ce nom affectent les formes les plus étranges. Le plus grand nombre ressemblent à des Potamides ou des Cérithes, quelques-unes à des Turritelles, d'autres, enfin, réunissent un assemblage de signes distinctifs qui ne se rapporte à rien de connu. En somme, ces Mélanidées forment un ensemble de formes spéciales à ce lac. C'est pour ce motif que je crois

1. J'ai adopté le nom de Nyassia, bien qu'il existât déjà un genre Nyassa (Hall, 1869) établi pour un fossile d'Amérique, parce que j'ai pensé qu'entre ces deux noms il y avait une différence assez notable pour qu'il ne puisse pas avoir confusion. Les auteurs ont bien admis le nom d'Hyalinia (Agassiz, 1837) pour distinguer les petits Zonites vitracés, lorsqu'il existait déjà le nom d'Hyalina (Studer, 1820) créé pour des Vitrines. On ne peut avoir deux poids et deux mesures. Ce qui est adoptable pour l'un doit l'ètre pour l'autre. 
convenable de les inscrire sous le nouveau'nom que je propose. Les formes nyassiennes sont si bizarres qu'il est difficile d'assigner à chacune d'elles une classification bien méthodique. Néanmoins, l'on peut les répartir en cinq groupes :

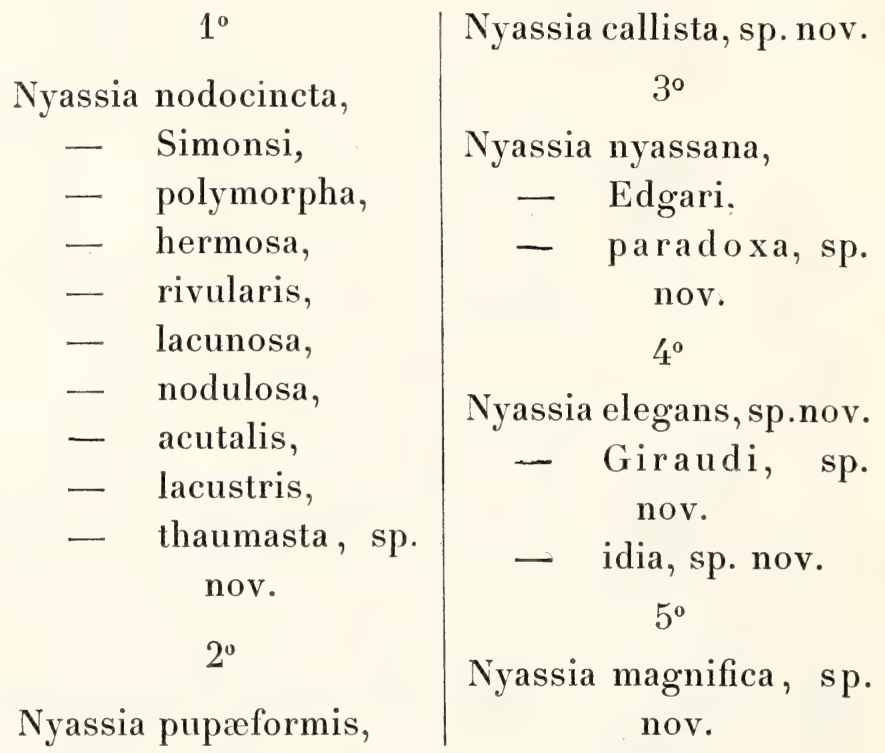

\section{NYASSIA NODOGINGTA}

Melania nodicincta, Dohrn, Shells Zambesi and lake Nyassa, in : Proceed. zool. Soc. Lond., 1865, p. 234; Smith, Shells Nyassa, in : Proceed. zool. Soc. Lond., 1877, p. 715, pl. Lxxv, fig. 11-12.

Coquille allongée-turriculée, à test solide, olivàtre, orné de fortes costulations transverses légèrement arquées et vigoureusement noduleuses, no- 
tamment le long de la suture, et disparaissant sur la partie moyenne du dernier tour pour faire place à huit ou dix sillons spiraux ${ }^{1}$. Spire très allongée, cylindrique-acuminée, à sommet lisse. Huit à dix tours à peine convexes, à croissance très lente, séparés par une suture ressemblant à une rainure profonde. Dernier tour égalant le tiers de la hauteur, plus convexe-arrondi à l'ouverture; celle-ci pyriforme, épanouie à la base, est entourée par un bord péristomal peu épais. - Haut., 17-20; diam., 8-9; - haut. ouv., 5 1/2-6 3/4 millim.

Espèce abondante sur les roseaux à l'extrémité nord du lac, à l'endroit où commence le Shiré.

\section{NYASSIA SIMONSI}

Melania Simonsi, Smith, Shells Nyassa, in : Proceed. zool. Soc. Lond., 1877, p. 713, pl. Lxxv, fig. 3 .

Coquille de grande taille (haut. 18; diam. $61 / 2$; haut. ouv. 6 millim.), pyramidale-allongée, assez ventrue au dernier tour, à test mince, transparent, blanchâtre, maculé de taches d'un rouge marron foncé, en séries interrompues, et orné de costulations assez semblables à celles de la nodocincta, sauf celles circumsuturales qui paraissent plus fortes et plus saillantes. Spire régulièrement acuminée, mais d'une facon plus courte que celle

1. D'après les figures données par Smith, les nodosités semblent s'étendre jusqu'à la base du dernier tour. Ce caractère ne concorde pas avec la description de Dohrn. Ces figures doivent être fautives. 
de la nodocincta. Neuf tours méplans-tectiformes. Ouverture ovale très anguleuse au sommet.

NYASSIA POLYMORPHA

Melania polymorpha, Smith, Shells Nyassa, in : Proceed. zool. Soc. Lond., 1877, p. 714, pl. Lxxv, fig. 4 (seulement).

Sous le nom de polymorpha, le $\mathrm{D}^{\mathrm{r}}$ Edgar Smith a confondu, à mon sens, six formes différentes et très distinctes les unes des autres.

Le type polymorpha, tel qu'il est figuré, est une coquille de belle taille (haut. 17 millim.), régulièrement acuminée, à costulations s'accusant par des sillons spiraux très émoussés, non noduleux, sauf le long de la suture, et caractérisée par une ouverture ovalaire, très anguleuse au sommet, arrondie et bien dilatée inférieurement.

NYASSIA HERMOSA

Melania polymorpha, Smith, Shells Nyassa, in : Proceed. zool. Soc. Lond., 1877, pl. Lxxv, fig. 5 (seulement).

Cette forme, d'une taille moindre (haut. 1213 millim.), se distingue de la polymorpha type par sa forme moins régulièrement acuminée, par son dernier tour allant en s'atténuant à la base, par suite du maximum de convexité plus supérieur chez lui que chez la polymorpha, par son ouverture plus étroite, plus anguleuse au sommet, moins dilatée à la base, par ses costulations noduleuses. 


\section{NYASSIA RIVULARIS}

Melania polymorpha, Smith, Shells Nyassa, in : Proceed. zool. Soc. Lond., 1877, pl. Lxxv, fig. 6 ( seulement).

Celte Nyassie diffère des deux qui précèdent par son test plus gros, plus trapu, moins bien acuminé, conservant, en effet, une certaine ventrosité presque jusqu'au sommet pour s'atténuer assez brièvement; par son dernier tour plus ventru, par cela même que le maximum de la convexité se trouve plus inférieur; par son ouverture plus arrondie, plus dilatée inférieurement, enfin, par son mode différent de costulations.

NYASSIA LACUNOSA

Melania polymorpha, Smith, Shells Nyassa, in : Proceed. zool. Soc. Lond., 1877, pl. Lxxv, fig. 7 ( seulement).

Chez cette Espèce (haut. 10 millim.), le test, entièrement recouvert de costulations noduleuses, est régulièrement acuminé ; le dernier tour parait bien développé ; l'ouverture, irrégulièrement ovalaire, offre supérieurement une angulosité prolongée, une partie médiane ample et une inférieure sensiblement atténuée, par suite de la grande courbure du bord columellaire, qui, de plus, à son extrémité, semble un tant soit peu tronqué. 
NYASSIA NODULOSA

Melania polymorpha, Smith, Shells Nyassa, in : Proceed. zool. Soc. Lond., 1877, pl. Lxxv, fig. 8 ( seulement).

Cette autre Nyassie (haut. 11 millim) est plus fortement noduleuse que la précédente; bien que de même forme, quoiqu'un peu moins ventrue, au point de vue de l'acumination, elle s'en distingue nettement par son ouverture d'un ovale subarrondi, à peine anguleux au sommet. Chez celle-ci, le bord columellaire, au lieu d'être courbe, descend obliquement d'une facon rectiligne, ce qui donne à l'ouverture une forme toute différente de la Lacunosa et des autres Nyassies précédentes.

\section{NYASSIA ACUTALIS}

Melania polymorpha, Smilh, Shells Nyassa, in : Proceed. zool. Soc. Lond., 1877, pl. Lxxv, fig. 10 (seulement).

Coquille (haut. 13 millim.) très allongée-acuminée pour sa grosseur. Test chargé de côtes noduleuses. Dernier tour, relativement petit, égalant juste le tiers de la hauteur (chez toutes les autres formes, confondues sous le nom de polymorpha, le dernier tour dépasse sensiblement le tiers). Tours à croissance plus serrée. Ouverture presque ronde. 
NYASSIA LACUSTRIS

Melania polymorpha, Smith, Shells Nyassa, in : Proceed. zool. Soc. Lond., 1877, pl. Lxxv, fig. 9 (seulement).

Cette petite Espèce (haut. 7 1/2 millimètres), fortement noduleuse, relativement assez ventrue pour sa taille et moins régulièrement acuminée que l'acutalis, est remarquable par son ouverture très portée du côté droit; celle-ci, fortement anguleuse au sommet, offre une rotondité assez régulière dans le reste de son contour.

NYASSIA THAUMASTA, sp. nov.

Coquille allongée-conoïde, brillante, d'une nuance cornée-olivâtre. Test assez épais, élégamment orné, autour de la suture, d'une ceinture de fortes nodosités, d'un ton plus pâle ressemblant à des perles, et, sur la surface des tours, de costulations très émoussées, peu perceptibles sur le dernier. Spire acuminée, tout en restant obtuse au sommet. Sept à huit tours plans, à croissance régulière, séparés par une rainure suturale. Dernier tour relativement ventru, arrondi, égalant le tiers de la hauteur. Ouverture arrondie, peu anguleuse au sommet. Péristome simple. - Hauteur 13 ; diam. 5 ; haut. ouv. 4 1/3 millim.

Plage de Karonga. 
NYASSIA PUPEFORMIS

Melania pupiformis, Smith, Shells Nyassa, in : Proceed. zool. Soc. Lond., 1877, p. 713, pl. Lxxv, fig. 13.

Coquille de forme pupoïde, cylindrique-allongée, fort peu acuminée, sauf vers les tours supérieurs. Test jaunacé, couvert de còtes noduleuses. Neuf tours peu convexes, à croissance lente. Dernier tour égalant le tiers de la hauteur. Ouverture ovalaire, anguleuse au sommet. Haut. 11 ; diam. $31 / 2$; haut. ouv. $31 / 2$ millim.

NYASSIA CALLISTA, sp. nov. (pl. in, fig. 1-2.)

Coquille de forme également pupoïde, mais à un degré moindre, plus longuement acuminée, assez épaisse, peu transparente, d'une teinte cornée avec deux bandes brunes, l'une supérieure au-dessous du renflement carénal, l'autre à la base du dernier tour. Test sillonné de costulations noduleuses très émoussées, présentant le long de la suture un renflement saillant, couronné de nodosités proéminentes. Spire allongée, acuminée, tout en conservant une certaine ventrosité relative jusqu'aux deux tiers de la hauteur, puis terminée par un sommet aigu. Neuf tours, les supérieurs plans-tectiformes, les autres renflés à la suture, puis à peine convexes. Croissance spirale régulière. Suture ressemblant à une rainure. Dernier tour égalant le tiers de la hauteur, légèremen 
concave en dessous du renflement supérieur, puis moyennement convexe. Ouverture assez oblique, ovale, peu anguleuse au sommet. Péristome simple. - Haut. 13; diam. 5; haut. ouv. 4-4 1/4 mill.

Cette Nyassie se distingue de la pupæformis par son test inférieurement plus ventru, allant en s'acuminant d'une façon plus régulière, ce qui lui donne une apparence un peu moins pupoïde, par son sommet plus conique, par ses costulations plus émoussées, et par son renflement suturo-carénal plus saillant, ainsi que par son ouverture plus régulièrement ovale et moins anguleuse au sommet.

\section{NYASSIA NYASSANA (pl. II, fig. 13-14).}

Melania Nyassana, Smith, Shells Nyassa, in : Proc. zool. Soc. Lond., 1877, p. 715, pl.' Lxxv, fig. 1 (seulement; la figure 2 se rapporte à l'espèce suivante).

Je donne la représentation d'un échantillon remarquable par son test orné de costulations très émoussées et par son renflement suturo-carénal couronné de nodosités. Chez le type, figuré par le docteur Smith, on ne remarque ni ces costulations, ni ces nodosités. Cette Espèce paraît abondante dans le lac.

\section{NYASSIA EDGARI}

Melania Nyassana, Var. Smith, Shells Nyassa, in : Proc. zool. Soc. Lond., 1877, pl. Lxxv, fig. 2 (seulement). 
Cette forme, qui ressemble tout à fait à une Turritelle, se distingue de la Nyassana par sa taille plus allongée, par sa spire plus cylindrique et plus lentement acuminée, par son test inférieurement non ventru, par sa croissance spirale plus lente, par son dernier tour bien moins grand, par son ouverture moins anguleuse au sommet et caractérisée, sur le côté columellaire, par un sillon assez prononcé.

NYASSIA PARADOXA, spec. nov. (pl. II, fig. 3-4.)

Coquille allongée, des plus singulières au point de vue de la forme. Le sommet ressemble à un dôme supporté par un tour (le cinquième), dont les costulations simulent une rangée de colonnes, puis, à partir de ce tour, la suture devient descendante, les tours se gonflent en une carène circumsuturale très proéminente, s'étranglent au milieu, pour redevenir convexes à la base.

Test transparent, très brillant, d'un blanc lactescent, orné d'une carène circumsuturale continue, sans nodosités, puis de costulations transverses très émoussées, enfin, à la base du dernier tour, de sillons spiraux. Spire allongée, scalariforme, en forme de dôme assez pointu au sommet. Huit tours : les quatre supérieurs exigus, lisses, tectiformes, à croissance lente; le cinquième costulé, relativement très développé, de plus, ventru; les autres, descendants, tous costulés, à croissance accélérée. Suture superficielle et horizontale jusqu'au sixième tour, puis, devenant profonde et très 
descendante. Dernier tour, grand, étranglé au milieu, convexe et relativement ventru inférieurement, enfin entouré supérieurement d'une très forte carène circumsuturale. Ouverture verticale, oblongue, très anguleuse au sommet. Péristome simple. - Haut., 11; diam., 3 ; haut. ouv., 4 millim.

Cette Nyassie est si singulière de forme et offre des caractères si bizarres, qu'elle ne peut être rapprochée d'aucune de ce lac.

NYASSIA ELEGANS, spec. nov. (pl. II, fig. 7-8.)

Coquille oblongue-pupoïde, à test assez épais, brillant, d'une teinte pâle cornée, lisse, sans costulations ni nodosités, sauf quelques vestiges noduleux le long de la suture sur les deux derniers tours. Spire oblongue, obtusément atténuée. Huit tours peu convexes, à croissance lente, séparés par une suture superficielle, devenant plus profonde en approchant de l'ouverture. Dernier tour dépassant un peu le tiers de la hauteur, médiocrement convexe. Ouverture verticale, ovale, anguleuse au sommet. Péristome simple. - Haut., 13; diam., 4 ; haut. ouv., 4 millim.

NYASSIA GIRAUDI, spec. nov. (pl. II, fig. 9-10.)

Cette Espèce, dédiée au voyageur Victor Giraud, se distingue de la Nyassia elegans par sa taille plus faible, par sa forme plus fluette, moins ventrue, moins régulièrement oblongue-allongée, par ses derniers tours marqués de costulations trans- 
verses très émoussées, par son ouverture oblongue plus étroite, plus anguleuse au sommet.

NYASSIA IDIA, spec. nov. (pl. II, fig. 11-12.)

Espèce caractérisée par une coquille allongéesubconoïde, à sommet obtus, à test blanc-cristallin brillant, transparent, costulé, seulement sur les tours médians, et lisse sur les deux derniers et sur les tours embryonnaires. Huit tours à croissance régulière, méplans ou à peine convexes, sans carène circumsuturale. Dernier tour égalant presque le tiers de la hauteur, d'abord méplan, puis devenant de plus en plus convexe en approchant de l'ouverture; celle-ci pyriforme, très anguleuse au sommet, est entourée par un bord péristomal simple. - Haut., 14; diam., 4; haut. ouv., 4 1/2 millim.

NYASSIA MAGNIFIGA, spec. nov. (pl. II, fig. 5-6.)

Coquille cylindrique, allongée, brièvement atténuée au sommet en forme de dôme, et arrondie inférieurement. Test assez épais, brillant, transparent, d'un corné pâle, orné sur les tours médians de fortes costulations émoussées et, le long de la suture, d'un gros renflement couronné de nodosités. Spire cylindriforme, à sommet obtus et brièvement atténué. Huit tours à croissance lente et régulière; les supérieurs lisses, méplans-tectiformes; les médians, renflés le long de la suture, un tant soit peu concaves puis convexes; enfin, le dernier, semblable aux médians, seulement bien 
convexe à l'ouverture; celle-ci subarrondie, faiblement anguleuse au sommet, est entourée par un péristome simple. - Haut., 12 ; diam., 4 ; haut. ouv., $33 / 4$ millim.

\section{NYASSELLA}

Ainsi que le nom l'indique, les Nyasselles sont des miniatures de Nyassies; ce sont de très petites coquilles oblongues-conoïdes, à test brillant, trans parent, lisse, ou costulé, ou noduleux, ou bien encore suturo-caréné, orné de flammules d'un rouge-marron très foncé, tantôt fulgurantes, tantôt en séries plus ou moins interrompues. Chez les Nyasselles, la spire, toujours acuminée, est régulière, et l'ouverture, de forme plus ou moins oblongue, offre toujours, à la base, un épanouissement-patulescent.

Les Nyasselles ont tout à fait l'apparence de Rissoa. L'une d'elles, la Nyassella formosa, a son extrémité apicale inclinée comme celle des Stylifer ovoideus ou subulatus.

Les formes de ce genre peuvent se répartir :

$1^{\circ}$ En Espèces costulées,

Nyassella Smithi, sp. nov.

- pulchra, id.

- arenaria, id.

- formosa, $i d$.

$2^{\circ}$ En Espèces lisses,

Nyassella episema, sp. nov.

- acuminata, id.

- Tayloriana, $i d$.

Bull. Sor. malac. France, VI. Juin 1889. - 2 
NYASSELLA SMITHI, sp. nov. (pl. I, fig. 1つ-16.)

Coquille allongée-fusiforme, acuminée à la partie supérieure, atténuée à la base, brillante, transparente, d'une teinte blanche-hyaline avec des taches rouge-marron foncées en séries très interrompues sur le dernier tour. Test élégamment costulé par de fortes côtes émoussées, noduleuses, notamment sur la ligne suturale, s'interrompant sur le milieu du dernier tour, où elles sont remplacées par deux sillons spiraux très obsolètes. Spire régulièrement acuminée-allongée, à sommet légèrement obtus. Six à sept tours méplanstectiformes, à croissance régulière, séparés par une suture ressemblant à une rainure profonde par suite du renflement noduleux suturo-carénal. Dernier tour n'atteignant pas la moitié de la hauteur, méplan-tectiforme jusqu'à la partie moyenne, puis allant en s'atténuant tout en conservant une certaine convexité. Ouverture presque verticale, oblongue, rétrécie à ses extrémités supérieure et inférieure, et largement épanouie à la base. Bord columellaire recto-descendant dans une direction inclinée de gauche à droite. Bord externe légèrement sinueux. Péristome presque aigu, faiblement crénelé-ondulé. Bords marginaux réunis par une faible callosité transparente. - Haut, 8; diam. 3 ; haut. ouv., 3 millim.

Cette belle espèce est dédiée au $D^{r}$ Edgar Smith, du British Museum. 
NYASSELLA PULGHRA, spec. nov, (pl. I, fig. 17-8.)

Coquille oblongue-acuminée, assez ventrue dans sa région inférieure, brillante, transparente, d'un blanc lactescent avec de petites taches rouge-marron très foncées en séries interrompues. Test costulé par des côtes très émoussées, peu noduleuses, disparaissant sur la partie moyenne du dernier tour, et pourvu le long de la suture d'un gros renflement noduleux formant saillie. Spire acuminée, tout en conservant un sentiment de convexité. Sept tours méplans, à croissance régulière, séparés par une rainure suturale profonde dominée par la saillie du renflement suturo-carénal. Dernier tour n'atteignant pas la moitié de la hauteur, convexe, non atténué à la base. Ouverture oblongue, anguleuse seulement au sommet, arrondie et développée à la base, enfin nettement épanouie-patulescente. Bord columellaire faiblement arqué. Péristome aigu, légèrement épaissi inférieurement. Callosité médiocre. - Haut. 8-9; diam. 3-3 1/2; haut. ouv. 4 millimètres.

Cette Espèce se distingue de la précédente par sa forme oblongue, non atténuée à la base, par sa spire non régulièrement acuminée, mais subconvexe-acuminée, par ses tours seulement méplans, non tectiformes, par son dernier tour plus ventru, plus convexe, non atténué à la base, par son ouverture développée et bien ronde inférieurement et non contractée comme celle de la Smithi, par son bord columellaire non rectiligne mais faiblement 
courbe; enfin, par ses costulations différentes et sans renflement suturo-carénal plus saillant.

NYASSELLA ARENARIA, spec. nov. (pl. II, fig. 17-18.)

Coquille de petite taille, de forme oblongue, obtusément acuminée, brillante, transparente, avec quelques petites maculatures rouge-marron disséminées cà et là. Test orné de côtes très émoussées, noduleuses seulement le long de la suture et à la partie moyenne du dernier tour, où les costulations disparaissent. Spire obtusément acuminée, à sommet gros, émoussé. Sept tours méplans, un tant soit peu subconvexes, à croissance régulière, séparés par une rainure suturale. Dernier tour égalant presque la moitié de la hauteur, avec un sentiment d'angulosité à la partie moyenne et atténué à la base. Ouverture verticale oblongue, un peu en forme de losange, présentant quatre angulosités : une supérieure aiguë, une inférieure obtuse et deux médianes, également obtuses, au sommet du bord columellaire et sur le côté externe. Bord columellaire rectiligne. Péristome simple, patulescent à la base. - Haut. 5 2/3-6; diam. 2 ; haut. ouv. 2 1/2 millim.

Cette Nyassie se distingue de la pulchra par sa taille moindre, par son mode différent de costulations, par la rareté de ses maculatures, par sa spire plus obtusément acuminée, par son sommet plus gros, par son dernier tour légèrement anguleux et notamment par son ouverture losangique à quatre angulosités. 
NYASSELLA FORMOSA, spec. nov. (pl. I, fig. 23-24.)

Coquille ventrue, de forme oblongue-acuminée, à sommet incliné à gauche, brillante, transparente, d'une nuance hyaline jaunâtre ou lactescente avec des taches rouge-marron très foncées en séries interrompues. Test costulé de côtes émoussées, noduleuses, seulement sur le renflement suturocarénal, et disparaissant à la partie moyenne. Spire acuminée, surmontée par deux ou trois tours embryonnaires, relativement grêles, styliformes et inclinés du côté gauche. Sept à huit tours; les supérieurs très exigus, lisses, à croissance régulière, méplans tout en étant un tant soit peu convexes. Suture enfoncée, par suite du renflement carénal. Dernier tour n'atteignant pas la moitié de la hauteur, assez ventru et convexe, tout en conservant à la partie supérieure une apparence méplane. Ouverture oblongue, anguleuse supérieurement, arrondie et bien épanouie-patulescente inférieurement. Péristome aigu, épaissi à la base. Callosité médiocre et transparente. - Haut. 11 ; diam. 4; haut. ouv. 4-5 millim.

La forme plus ventrue de cette Espèce, son mode différent de costulations, non moins que son sommet styliforme incliné à gauche, composé de tours exigus à croissance serrée, la différencieront toujours des précédentes. 
NYASSELLA EPISEMA, spec. nov. (pI. I, fig. 19-20.)

Coquille allongée-acuminée, de plus, scalariforme, brillante, peu transparente, assez épaisse, d'une teinte légèrement cornée avec des fulgurations rouge-marron très foncées. Test bien lisse, sans trace de costulations, pourvu, seulement le long de la suture, d'une carène lisse formant saillie et sans vestige de nodosités. Spire scalariforme, régulièrement acuminée, à sommet petit. Sept à huit tours méplans renflés supérieurement, à croissance régulière, séparés par une suture enfoncée. Dernier tour n'atteignant pas la moitié de la hauteur, méplan, néanmoins un tant soit peu concave en dessous du renflement carénal. Ouverture presque verticale, oblongue, épanouie à la base. Péristome aigu. Callosité faible. - Haut. 9 ; diam. 3 ; haut.; ouv. 3 millim.

Cette Nyasselle, qui n'a aucun rapport avec les Espèces qui précèdent, rappelle par sa coloration et sa spire scalariforme certaines Mitres de l'Océan indien.

NYASSELLA AGUMINATA, spec. nov. (pl. I, fig. 21-22.)

Coquille conique, régulièrement acuminée, assez ventrue inférieurement, brillante, substransparente, relativement épaisse, lactescente avec quelques maculatures rouge-marron foncées en séries très interrompues. Test lisse, paraissant, sous le foyer d'une forte loupe, comme chagriné. Spire allongée-conique, à sommet petit. Sept tours 
plans-tectiformes, à croissance régulière, séparés par une suture superficielle. Dernier tour n'atteignant pas la moitié de la hauteur, convexe à la base. Ouverture verticale, pyriforme, patulescente inférieurement. Bord externe arqué en avant. Péristome aigu, relativement épaissi en dedans. - Haut. 8 ; diam. 3 ; haut. ouv. 3 millim.

Cette Espèce, par sa forme acuminée, bien conique, par son test lisse, par sa coloration, par sa suture superficielle, par son manque de renflement suturo-carénal, par son ouverture pyriforme, etc... ne peut être non plus confondue avec les Nyasselles précédentes.

NYASSELLA TAYLORIANA, spec. nov. (pl. 1, fig. 25-26.)

Coquille fusiforme, acuminée supérieurement, atténuée inférieurement, brillante, transparente, bien qu'assez épaisse, d'un blanc lactescent tirant un peu sur une nuance jaunacée, avec quelques maculatures rouge-marron foncées en séries interrompues. Test lisse, avec un sentiment de renflement carénal le long de la suture. Spire allongée, régulièrement acuminée, à sommet un peu obtus. Sept tours plans, à croissance régulière, séparés par une suture assez prononcée. Dernier tour égalant la moitié de la hauteur, faiblement convexe, atténué à la base. Ouverture verticale oblongueallongée, contractée en haut et en bas, bien épanouie à la base. Bord externe arqué en avant. Péristome aigu, épaissi à l'endroit de l'épanouissement. 
Callosité transparente. - Haut. 9-10; diam. 4; haut. ouv. 4 1/2-5 millim.

Cette Espèce, à laquelle j'attribue le nom de M. John W. Taylor, directeur du Journal of Conchology d'Angleterre, et membre de la Société malacologique de France, est encore une Nyasselle si différente de toutes les autres, qu'il est impossible de la confondre avec aucune d'elles.

\section{MJCRONYASSIA}

Les formes, comprises dans cette coupe générique, sont de petits Mélaniens à coquille écourtée, trapue, à spire brièvement conique, surmontée d'un sommet mamelonné, caractérisé, en outre, par un test brillant, assez transparent, d'une teinte uniforme cornée ou olivâtre, et orné, le long de la suture, notamment sur le dernier tour, d'un renflement carénal, toujours très accusé, tantôt simple, tantôt noduleux, et sur la surface de ce tour, de nodosités plus ou moins accentuées, nodosités toujours supérieures et ne dépassant pas la partie médiane.

Les Espèces de cette coupe sont de fort petite taille; la plus grande ne dépasse pas 8 millimètres. Elles peuvent être réparties :

$1^{\circ}$ En Espèces à spire normale, c'est-à-dire en Espèces chez lesquelles les tours supérieurs s'accroissent régulièrement, sans être par trop disproportionnés en taille et en volume avec le dernier tour. 
Micronyassia turritospira.

- Smithi (nov. spec.).

$2^{\circ}$ En Espèces à spire anormale, c'est-à-dire en Espèces chez lesquelles les tours supérieurs sont en notable disproportion avec le dernier.

A. Spire conique, relativement allongée.

Micronyassia egregia.

- eximia, nov. spec.

- Giraudi. id.

B. Spire très courte, tectiforme.

Micronyassia singularis, nov. spec.

\section{MICRONYASSIA TURRITOSPIRA}

Melania turritispira (pars), Smith, Shells Nyassa, in : Proc. zool. Soc. Lond., 1877, p. 713, pl. Lxxxv, fig. $14^{1}$ seulement (la figure 15 représente l'egregia).

Petite Espèce de forme ovale-conoïde, d'une teinte pâle, ornée d'une carène saillante circumsuturale pourvue de grosses nodosités, et, de plus, de larges côtes obsolètes, très émoussées, plus ou moins noduleuses, s'évanouissant, sur le dernier tour, un peu au-dessous de la région moyenne, pour faire place à des sillons spiraux. Spire relativement allongée, bien conique, à sommet lisse et gros. 7 à 8 tours méplans-tectiformes, renflés le long de la suture.

1. Très grossie. 
Ouverture subovalaire, très anguleuse au sommet, assez projetée du côté droit et entourée par un bord péristomal assez épais. - Haut., 8; diam., 3 1/2. - Haut. ouv., 3 millim.

MICRONYASSIA SMITHI (pl. I, fig. 3-4)

Coquille oblongue-ovoïde, de forme obèse, brillante, transparente, d'une nuance pâle hyalino-cornée. Test orné de grosses côtes émoussées, peu nombreuses, disparaissant à la région médiane du dernier tour, vaguement noduleuses, sauf sur le renflement carénal circumsutural, où les nodosités forment de fortes saillies obsolètes. Spire en dôme allongé, obtusément subaigu. Sommet lisse, moyennement mamelonné. 7 tours convexes, à croissance peu accélérée, séparés par une suture ondulée et relativement enfoncée par suite du renflement noduleux circumsutural. Dernier tour assez ventru, lisse inférieurement, égalant la moitié de la hauteur. Ouverture verticale, subarrondie. Columelle courte, bien arquée; péristome peu épais, presque aigu du côté externe, et légèrement patulescent à la base. Bords marginaux réunis par une forte callosité. Haut., 8; diam. 4 ; haut. ouv., 4 millim.

Cette Micronyassie, à laquelle j'attribue le nom du $\mathrm{D}^{\mathrm{r}}$ Edgar Smith, se distingue très nettement de la turritospira, par sa forme obèse, plus ventrue, par sa spire non conique-pyramidale, mais conoïde en forme de dôme allongé, par ses costulations différentes, par son ouverture presque ronde, non an- 
guleuse au sommet, par son dernier tour égalant la moitié de la hauteur, plus ventru, lisse à sa base et non sillonné de stries spirales.

Comme forme, cette Espèce ressemble beaucoup à l'Alvania rufilabrum de la Méditerranée.

\section{MICRONYASSIA EGREGIA}

Melania turritispira(alt. pars), Smith, Shells Nyassa, in : Proceed. zoolog. Soc. London, 1877, pl.Lxxxv, fig. $15^{1}$, seulement (la figure 14 représente le type turritospira).

Coquille de très petite taille (6 mill.), offrant à peu près le même mode de costulations que celui de la turritospira, avec laquelle le $\mathrm{D}^{\mathrm{r}}$ Smith l'a confondue, mais en différant essentiellement par sa spire écourtée, brièvement conique, complètement en disproportion avec le dernier tour qui est relativement énorme et dépassant la moitié de la hauteur; par son ouverture plus anguleuse au sommet et de forme différente ; par son renflement circumsutural plus fortement noduleux et plus saillant; par sa taille moindre, sa forme plus trapue et plus ventrue.

MIGRONYASSIA EXIMIA (pl. 1, fig. 1-2)

Coquille oblongue-conoïde, brillante, transparente, d'une teinte hyalino-cornée, un tant soit peu marron. Test élégamment orné de grosses côtes émoussées, faiblement obliques, disparaissant sur

1. Figure très grossie. 
la région médiane du dernier tour, non noduleuses, sauf sur le renflement carénal circumsutural, qui est saillant et relativement volumineux. Spire brièvement conique, délicate, surmontée d'un sommet mamelonné. 6 tours, les 3 supérieurs lisses et convexes, le quatrième tectiforme, les deux derniers fortement renflés le long de la suture qui paraît, par cela même, enfoncée. Dernier tour égalant la moitié de la hauteur, fortement caréné le long de la suture, puis méplan jusqu'au niveau de la zone de disparition des côtes, enfin, lisse et s'atténuant, tout en conservant une certaine convexité. Ouverture peu oblique, oblongue, anguleuse au sommet. Péristome aigu, faiblement patulescent à la base. Bords marginaux réunis par une callosité d'une épaisseur médiocre. - Haut. 5-6; diam. 3; haut. ouv. 2 1/2 à 3 mill.

Le mode des costulations, la délicatesse de la spire, le mamelon apical, la forme de l'ouverture, non moins que la taille du dernier tour qui ne dépasse pas la moitié de la hauteur (c'est l'inverse chez l'Espèce précédente), feront toujours distinguer facilement l'eximia de l'egregia.

MICRONYASSIA GIRAUDI (pl. ।, fig. כั-6)

Coquille de forme oblongue-conoïde, tout en étant scalariforme à partir de l'avant-dernier tour, par suite de l'exagération du renflement carénosutural. Test brillant, hyalin, presque lisse, sauf des apparences de quelques gibbosités sur la région moyenne du dernier tour et sauf quelques 
soupçons de nodosités sur la carène. Spire courte, conique, tout à fait en disproportion de volume avec le reste de la coquille; sommet mamelonné ; 6 tours irréguliers: l'embryonnaire gros, en forme de mamelon, le deuxième étroit, le troisième convexe, très développé, le quatrième étroit, enfin les deux derniers très grands, avec une forte carène supérieure donnant lieu à une étroite zone circumsuturale méplane. Dernier tour dépassant un peu la moitié de la hauteur, légèrement concave au-dessous de la carène, puis faiblement convexe. Ouverture peu oblique, oblongue, contractée supérieurement et très anguleuse. Péristome aigu, subpatulescent à la base. Callosité très mince. Haut. 5 ; diam. $21 / 2$; haut. ouv. 3 millim.

Cette Espèce, remarquable par l'exiguïté de sa spire, par l'exagération du renflement carénosutural qui donne à cette Micronyassie un aspect scalariforme, par l'irrégularité de son mode de croissance, etc..., ne peut être confondue avec aucune autre. Elle est dédiée au célèbre voyageur Victor Giraud, qui l'a découverte.

MICRONYASSIA SINGULARIS (pl. I, fig. 7-8)

Coquille relativement très grosse pour sa taille, de forme irrégulièrement ovalaire, surmontée d'une spire écrasée, ressemblant à un couvre-chef conique-tectiforme. Test assez épais, tout en restant transparent, d'une teinte pâle cornée-olivâtre, orné d'une belle carène lisse circumsuturale, et, sur le dernier tour, de vestiges de nodosités très 
émoussées. Spire très courte, écrasée, coniquetectiforme. Sommet gros. Six tours, dont les supérieurs très exigus, méplans-tectiformes, le dernier très grand, faiblement concave au-dessous de la carène, puis convexe, dépassant un peu la moitié de la hauteur. Ouverture presque verticale, subarrondie, à peine anguleuse supérieurement. Péristome aigu, faiblement encrassé à la base. Callosité médiocre. - Haut. 6 ; diam. 4 ; haut. ouv. 3 1/2 millim.

Cette singulière Espèce, si différente de toutes les Micronyassies par sa spire en forme de couvercle ou de toit écrasé, par sa carène lisse et continue, par son diamètre énorme comparativement à sa hauteur, est une des formes les plus remarquables du lac Nyassa.

\section{NYASSOMELANIA}

Les Espèces de cette dernière coupe générique sont des formes allongées, à sommet souvent tronqué, pourvues d'un test blanc, transparent, presque vitrinoïde, sans coloration et sans striations bien apparentes.

Les trois Nyassomélanies, qui me sont connues, me paraissent des formes aberrantes très difficiles à classer, qui, quoique ne possédant aucun caractère bien saillant, ne peuvent néanmoins être comprises que dans la grande famille des Mélanidées. 
NYASSOMELANIA LEIA (pl. I, fig. 9-10)

Coquille limnéiforme, oblongue-allongée, à test assez épais, blanc, lisse et transparent. Spire allongée: allant en s'atténuant et offrant, entre le quatrième et le cinquième tour, un étranglement assez notable qui donne à la coquille un cachet tout particulier. Six tours convexes, à croissance rapide, un peu irrégulière, séparés par une suture profonde. Dernier tour convexe n'atteignant pas la moitié de la hauteur. Ouverture faiblement oblique, oblongue, anguleuse supérieurement. Péristome sensiblement épaissi, patulescent à la base. Callosité assez forte. Haut., 12; diam. 4; haut. ouv., 5 millim.

NYASSOMELANIA TRUNCATELLIFORMIS (pl. 1, fig. 11-12)

Coquille troncatelliforme, cylindrique, à test sensiblement épais, blanc, lisse et subtransparent. Spire allongée, de forme cylindrique, allant faiblement en s'atténuant, et toujours tronquée supérieurement. Tours, au nombre de cinq seulement (les supérieurs manquent constamment), légèrement convexes, à croissance régulière, séparés par une suture étroite, profonde, ressemblant à une rainure. Dernier tour relativement petit, convexe. Ouverture faiblement oblique, oblongue, épanouie à la base. Péristome simple, un peu épaissi inférieurement. Bords marginaux réunis par une assez forte callosité. - Haut., 11 ; diam., 3; haut. ouv., 3 1/2 millim. 
Cette Espèce, grâce à sa forme cylindrique et à son apparence troncatelliforme, est-si distincte de la précédente et de la suivante, qu'elle ne peut être confondue ni avec l'une ni avec l'autre.

NYASSOMELANIA LÆVIGATA (pl. I fig. fig. 13-14)

Coquille allongée, de forme turriculée, tout en étant acuminée. Test assez épais, blanchâtre, subtransparent, lisse, bien qu'offrant sur le milieu des tours des vestiges à peine perceptibles de renflements nodosiformes. Spire allongée, lentement acuminée, à sommet tronqué. Tours, au nom bre de huit (les supérieurs manquent), à peine convexes, à croissance lente, serrée et régulière, séparés par une suture bien marquée. Dernier tour peu développé en hauteur, mais relativement plus convexe que les autres et même assez ventru vers l'ouverture; celle-ci faiblement oblique, ovalaire, sensiblement épanouie à la base, est entourée par un bord péristomal simple, dont les extrémités sont réunies par une assez forte callosité. Haut., 16; diam., 5; haut.; ouv., 5 millim.

Par sa forme conoïdéo-turriculée, cette Espèce ne peut être rapprochée ni de la leia, ni de la truncatelliformis. 
MM. Dohrn ${ }^{1}$, Frauenfeld ${ }^{2}$, Lea ${ }^{3}$, Martens ${ }^{4}$ et Smith ${ }^{5}$ sont les seuls auteurs, à ma connaissance, qui ont fait connaitre les Fispèces du lac Nyassa. Ces Espèces, y compris celles que je viens de décrire, atteignent le chiffre de 64, chiffre bien minime pour la faune d'un aussi grand lac, offrant un développement de côtes de plus de 1500 kilomètres.

Voici les espèces.

\section{GASTEROPODA INOPERCULATA}

\section{PULMOBRANCHIATA}

\section{LIMNEIDE}

1. Limnæa natalensis, Krauss, Moll. Sudafr., p. 85, pl. v, fig. 15. 1848.

2. Physa Nyassana. Smith, Shells Nyassa, in :

1. List of the Land and freshwater shells of the Zambesi and lake Nyassa, collected by John Kirk. In : Proceed. zool. Soc. London, 1885 , p. 231 à 234 .

2. Description of seven new species of the genus Vivipara, in : Proceed. zool. Soc. London, 1865, p. 658-659.

3. In : Proceedings of the academy of nat. sc. of Philadel phia, avril 1864 - et, in : Journal acad. nat. sc. of Philadelphia, VI, 1866.

4. Verzeichniss der von Prof. Peters in Mozambique gesammelten Land und susswasser-Mollusken, in : Malakozoologischen Blätter, 1859, p. 211-221.

5. On the shells of lake Nyassa; in : Proceed. zool. Soc. London, 1877, p. 712-722. 2 planches.

Bull. Soc. malac. France. VI. Juin 1889. - 3 
Proceed. Zool. Soc. Lond., 1877, p. 717, pl. Lxxv, fig. 16-17.

3. Physa succinoides, Smith (Loc. sup. cit.), p. 718, pl. lxxv, fig. 19-20.

4. Physopsis africana, Krauss, Moll. Sudafr., p. 85, pl. v, fig. 4. 1848.

\section{GASTEROPODA OPERCULATA}

\section{BRANCHIATA}

\section{PALUDINIDE}

5. Vivipara polita, Frauenfeld, nam. Palud, in : Verhandl. Zool. gesellsch. Wien, 1865. p. 669. (Paludina polita, Frauenfeld, in : Verh. Zool. ges. Wien, 1862, p. 1162.)

6. Vivipara Jeffreysi ${ }^{1}$, Frauenfeld, Zool. Miscell. IV, in : Verhandl. Zool. ges. Wien, 1865, p. 532, pl. xхıг.

7. Vivipara capillata, Frauenfeld, Zool. Miscell., IV, in : Verh. zool. Ges. Wien., 1865, p. 533, pl. xxı. - Grandidier, in : Bull. Soc. malac. Fr., IV, 1887, p. 190. - (Pars), Smith, in : Proc. zool. Soc. Lond., 1877, p. 717, pl. Lxxv, fig. 3 (seulement), la figure 4 doit être rapportée à la Vivip. Smithi.

8. Vivipara Robertsoni, Frauenfeld (loc. sup. cit.), p. 533, pl. xxII. - Smith, in Proceed. zool. Soc. Lond., 1877, p. 717, pl. pl. Lxxv, fig. 5-6.

1. Non Paludina Jeffreysi de Smith, 1877. 
- Grandidier, in : Bull. Soc. malac. Fr., IV, 1887, p. 190.

9. Vivipara Simonsi, Bourguignat, 1888 (Palud. Jeffreysi [non Frauenfeld, 1865] de Smith, in : Proc. zool. Soc. Lond., 1877, pl. Lxxiv, fig. 2 (seulement). - La vraie Vivipara Jeffreysi de Frauenfeld (in : Verh. zool. ges. Wien, 1865, pl. xxII) est une Espèce à spire courte, à tours supérieurs non étagés, à l'avant-dernier tour excessivement gros et ventru, à ombilic plus ouvert, Espèce qui ne ressemble point à la soidisant Vio. Jeffreysi que Smith a fait représenter pl. Lxxiv, fig. 2 (Proceed. zool. Soc. Lond., 1877). Quant à la figure 1, présentée également sous le nom de Jeffreysi, ce n'est ni la Jeffieysi de Frauenfeld, ni la Simonsi, Bourguignat [Jeffreysi de Smith], c'est la Vivipare suivante :

10. Vivipara Smithi, Bourguignat, 1888. Cette Espèce que Smith a fait représenter sur la pl. Lxxiv (Proc. zool. Soc. Lond., 1877) sous le nom erroné de Pal. Jeffreysi, fig. 1, et de Paludina capillata [non Frauenfeld], fig. 4 , est une Espèce de petite taille, trapue, écourtée, à très large ouverture sensiblement développée du còté externe. Cette forme n'a pas de rapport, soit avec les Vivip. Jeffreysi et capillata de Frauenfeld, soit avec la Vivip. Simonsi, citée ci-dessus, comme l'on peut s'en convaincre par l'examen attentif des figures.

11. Amnicola Stanleyi, Bourguignat, 1888 (Bythi- 
nia Stanleyi, Smith), in : Proc. zool. Soc. Lond., 1877, pl. Lxxv, fig. 21 (seulement). La figure 22 représente une forme toute différente, qui est la suivante :

12. Amnicola nyassana, Bourguignat, 1888. (Bythinia Stanleyi [altera pars], Smith, in : Proceed. zool. Soc. Lond., 1877, pl. Lxxv, fig. 22.)

\section{AMPULLARID E}

13. Ampullaria gradata, Smith, in : Proc. zool. Soc. Lond., 1881, p. 289, pl. xxxıI, fig. 22.

14. Meladomus affinis, Bourguignat, 1888 (Lanistes affinis, Smith, Sh. Nyassa in : Proceed. zool. Soc. Lond., 1877, p. 716, pl. Lxxiv, fig. 7, et in : Proceed. zool. Soc. Lond., 1881, p. 290, pl. xxxiv, fig. 23). Cette figure donne la représentation d'un échantillon beaucoup plus fort que le type. Smith, du reste, fait observer qu'il n'avait à sa disposition qu'un échantillon de petite taille lorsqu'il a établi cette espèce.

15. Meladomus ovum, Bourguignat, Moll. Égypte, Abyss., p. 36, 1879. (Ampullaria ovum, Peters, in : Arch. f. naturg., I, 1835, p. 315. - Lanistes ovum de Dohrn, 1865 ; Pfeiffer, 1866; Smith, 1877.)

16. Meladomus purpureus, Bourg., Moll. Égypte, Abyss., p. 35, 1879. (Ampullaria purpurea, Jonas, in : Arch. f. naturg., p. 242, pl. x, fig. 1, 1839. - Bulimus tristis de Jay, 1839, Meladomus olivaceus [non Sowerby], $H$. et A. Adams, 
1854, et Lanistes purpureus de Dohrn, Pfeiffer, Smith, etc.)

17. Meladomus solidus, Bourg., 1884, et Grandidier, Moll. Ousagh, in : Bull. Soc. mal. Fr., IV, 1887, p. 191. (Lanistes solidus, Smith, Sh. Nyassa, in : Proc. zool. Soc. Lond., 1877, p. 716, pl. Lxxiv, fig. 10-11.)

18. Meladomus nyassanus, Bourg., Moll. Égypte, Abyss., p. 39, 1879. (Lanistes nyassanus, Dohrn, Moll. Zamb., in : Proc. zool. Soc. Lond., 1865, p. 233.)

\section{MELANIDA}

Dans cette famille se trouvent comprises les 36 espèces, dont je viens de parler, appartenant aux genres Melania (1), Nyassia (19), Nyassella(7), Micronyassia (6) et Nyassomelania (3). - Je ne rappelle ici ces Espèces en bloc que pour mémoire. (Espèces de 19 à 54.)

\section{ACEPHALA \\ LAMELLIBRANCHIATA.}

\section{SPHARIDE}

55. Corbicula nyassana, Bourg., 1888. (Cyrena radiata [non Parreyss] Smith, Sh. Nyassa, in : Proceed. zool. Soc. Lond., 1877, p. 718.)

56. Corbicula astartina Prime, cat. Corbic., in : American Journal Conch. (Cyrena astartina,Martens, in : Malak., Blätter, 1859, p. 219, pl. III, fig. 6-7.) 
57. Corbicula astartinella, Bourg., 1888. Spec. nov. de Karonga.

58. Corbicula Giraudi, Bourg., 1888. Spec. nov. de Karonga.

\section{UNIONIDE}

59. Unio nyassanus, Bourg.(Unio nyassaensis, Lea, in : Proceed. acad. nat. sc. Philadelphia, 1864, p. 108, et in : Journ. acad. nat. sc. Phil., VI, 1866, p. 33, pl. xiı, fig. 32.)

60. Unio Kirki, Lea, in : Proc. acad. nat. sc. Phil., 1864, p. 108, et in : Journ. acad. nat. sc. Phil., VI, 1866, p. 32, pl. xII, fig. 30.

61. Unio aferulus, Lea, in : Proceed. acad. nat. sc. Phil., 1864, p. 109, et in : Journ. acad. nat. sc. Phil., VI, 1866, p. 34, pl. xIII, fig. 34.

Smith (Sh. Nyassa, in : Proc. zool. Soc. Lond., 1877, p. 719), par suite d'une erreur évidente d'appréciation, a réuni en une Espèce, sous le nom impropre de nyassaensis, ces trois Unios si caractérisés et si distincts les uns des autres, et ce qu'il y a de plus extraordinaire, cet auteur a fait figurer, parmi la série des Grandidieries (Shells Tangan., in : Proc. zool. Soc., Lond., 1881, pl. xxxıv, fig. 34 seulement, sous le nom d'Unio nyassaensis, un véritable Unio du Nyassa, différent des trois publiés par Lea. Cet Unio est le suivant :

62. Unio Hermosus, Bourg, 1888. Sp. nov. 
IRIDINID E

63. Spathella nyassana, Bourg., 1888. (Spatha Nyassaensis, Lea, in : Proc. acad. nat. sc. Phil., 1864, p. 109, et in : Journ. acad. nat. sc. Phil., p. 36, pl. xIII, fig. 33, 1866.)

64. Burtonia alata, Bourg., 1888. (Spatha alata, Lea [loc. sup. cit.], p. 109, 1864, et VI, 1866, p. 35, pl. xII, fig. 31.)

Ce qui frappe à première vue c'est, à l'exception de deux formes, l'Ampullaria gradata et le Meladomus nyassanus, de taille médiocre, c'est, dis-je, l'exiguïté de toutes les espèces. Toutes sont de petite taille; les acéphales mêmes sont singulièrement exigus. Ainsi, le plus grand des Unios ne dépasse pas 30 millimètres. La Spathella et la Burtonia sont également, à ma connaissance, les plus petites formes de ces genres. Il y a une opinion accréditée dans bien des ouvrages, c'est que les Espèces prennent un plus grand développement dans une vaste étendue d'eau que dans une petite. Cette opinion, à mon sens, est fausse. On en voit la preuve par cet immense lac de $30000 \mathrm{ki}$ lomètres de superficie. Il y a une autre cause, cette cause réside dans la grande crudité des eaux de ce lac, eaux sans parties calcaires, puisqu'elles ne laissent à l'ébullition aucune trace de sédiment sur la paroi des chaudières. J'ai toujours reconnu que les eaux les plus saturées de calcaire, je dirai même les plus impures, c'est-à-dire les plus chargées de 
détritus, étaient celles qui recélaient les plus belles et les plus fortes Espèces.

Les Pulmobranches nyassiens, représentés par une Limnée, deux Physes et une Physopside, sont des formes fluviales dont les analogues se rencontrent dans toutes les régions orientales du continent du Cap au bassin nilotique. Ces Espèces n'ont rien de particulier.

Les Vivipares n'ont pas de rapport avec la nombreuse série des Unicolor, si répandue en Afrique, ni avec les Paludinidées du Tanganika, mais elles offrent une certaine tendance de similitude, aux points de vue de la forme et du galbe, avec diverses Vivipares du nord de la Chine; en tout cas, ces Espèces possèdent un cachet plus septentrional et moins africain que celui qu'on remarque sur les congénères du Tanganika.

Les Ampullaridées, à l'exception du Meladomus nyassanus, spécial au lac, se composent de formes nettement africaines, formes répandues dans toutes les régions orientales, depuis le pays des Comalis jusqu'au Transwaal.

Les Mélanidées, bien que présentant pour la plupart des formes affines à celles des Potamides, des Cérithes et des Rissoas, sont toutes petites et possèdent à un degré moindre l'aspect thalassoïde si remarquable des Mélaniens du Tanganika.

Parmi les Acéphalés, les Corbicules, la Spathelle et la Burtonie, toutes de taille exiguë, portent le cachet de la faune de ce continent, tandis 
que les Unios, caractérisés par de petites formes subarrondies, à test épais, à charnière très volumineuse, munie d'une grosse cardinale attenante à une latérale très courte, n'ont rien d'africain. C'est en Amérique et en Asie que l'on rencontre de nombreuses Espèces offrant à peu près le même aspect que celui des Unios nyassiens.

En somme, l'ensemble de la faune dénote une faune distincte et tout à fait différente, au point de vue des formes, de celle du Tanganika. Malgré l'assimilation erronée, faite par le $\mathrm{D}^{\mathrm{r}}$ Smith, d'une Grandidierie avec un Unio du Nyassa, et à l'exception de l'inévitable Melania tuberculata, que l'on retrouve partout, pas une espèce n'est commune à ces deux lacs.

Voici, du reste, comme terme de comparaison, la liste exacte des nombreuses Espèces tanganikiennes, connues à présent, grâce aux recherches de MM. Victor Giraud, Léopold Joubert, et des RR. PP. missionnaires :

\section{GASTEROPODA INOPERCULATA}

\section{PULMOBRANCHIATA}

\section{LIMNEIDE}

1. Limnæa Jouberti, Bourg., Malac. Tang., pl. I, fig. 23, 1888.

2. Limnæa Laurenti, Bourg., Mal. Tang., pl. I, fig. 21-22.

3. Limnxa Alexandrina, Bourg., Mal. Abyss., p. 92 el 93, fig. 95 et $96,1883$. 
4. Limnæa Lavigeriana, Bourg., Mal. Tang., pl. I, fig. 18-19.

5. Limnxa africana, Ruppell, in : Bourg., Malac. Abyss., p. 95 et 126, fig. 99, 1883.

6. Limnæa Debaizei, Bourg., Mal. Tang., pl. I, fig. 20.

7. Physa Randabeli, Bourg., Mal. Tang., pl. I, fig. 26-27.

8. Physa Coulboisi, Bourg., Mal. Tang., pl. I, fig. 24-25.

PLANORBIDE

9. Planorbis sudanicus, Martens, in : Malak. Bl., p. 35, 1870. - Bourg., Mal. Tang., pl. 1, fig. 13-15.

10. Planorbis tanganikanus, Bourg., Mal. Tang., pl. I, fig. 16-17.

11. Planorbis adowensis, Bourg., Moll. Égypte, Abyss., p. 11, 1879, et Mal. Tang., pl. I, fig. 1-4.

12. Planorbis Monceti, Bourg., Mal. Tang., 1888.

13. Planorbis Lavigerianus, Bourg., Mal. Tang., pl. I, fig. 5-8.

14. Planorbis Bridouxianus, Bourg., Mal. Tang., pl. I, fig. 9-12.

15. Planorbula tanganikana, Bourg., M..l. Tang. (Seginentina [Planorbula] alexandrina, var. tanganyicensis, Smith., 1881). 


\section{GASTEROPODA OPERCULATA}

\section{BRANCHIATA}

PALUDINIDE

16. Neothauma tanganikanum, Grandidier, in : Bull. Soc. Mal. Fr. 11, 1885, p. 163, et Bourg., Mal. Tang., 1888, pl. II, fig. 1.

17. Neothauma Bridouxianum, Grandidier, in : Bull. Soc. Mal. Fr. 11, 1885, p. 163, et Bourg., Mal. Tang., pl. II, fig. 2-4.

18. Neothauma Giraudi, Bourg., Moll. Giraud Tang., p. 27, 1885, et Mal. Tang., pl. II, fig. 5-6.

19. Neothauma Servainianum, Grandidier, in : Bull. Soc. Mal. Fr. 11, 1885, p. 163, et Bourg., Mal. Tang., pl. III, fig. 2-3.

20. Neothauma bicarinatum, Bourg., Moll. Giraud Tang., p. 28, 1885, et Mal. Tang., pl. III, fig. 1.

21. Neothauma Jouberti, Bourg., Mal. Tang., pl. III, fig. 4-5.

22. Neothauma euryomphalus, Bourg., Mal. Tang., pl. II, fig. 7-8.

23. Neothauma Vysseri, Bourg., Mal. Tang., pl. III, fig. 6.

24. Vivipara Brincatiana, Bourg., Mal. Tang., pl. IV. fig. 1.

25. Vivipara Bridouxiana, Bourg., Mal. Tang., pl. iv, fig. 2.

26. Cleopatra Guillemeti, Bourg., Esp. nouv. et 
g. nouv. Oukér. et Tang., p. 6, 1885, et Mal. Tang., pl. Iv, fig. 4.

27. Cleopatra Jouberti, Bourg., Mal. Tang., pl. Iv, fig. 3.

28. Bythinia multisulcata, Bourg., Mal. Tang., pl. III, fig. 7-8.

29. Bridouxia Giraudi, Bourg., Moll. Giraud. Tang., p. 30, 1885, et Mal. Tang., pl. Iv, fig. 5-7.

30. Bridouxia Villeserriana, Bourg., Loc. sup. cit., p. 30, 1885, et Mal. Tang., pl. Iv, fig. 8-10.

31. Bridouxia costata, Bourg., Loc. sup. cit., p. 31, 1885, et Mal. Tang., pl. Iv, fig. 11-13.

32. Bridouxia Reymondi, Giraud, in : Bourg., Loc. sup. cit., p. 32, 1885, et Mal. Tang., pl. Iv, fig. 14-18.

33. Baizea Giraudi, Bourg., Loc. sup. cit., p. 34, 1885, et Mal. Tang., pl. Iv, fig. 17-19.

34. Spekia zonata, Bourg., Moll. Égypte, Abyss., p. 28, 1879, et Moll. Giraud, Tang., p. 37, 1885, et Mal. Tang., pl. Iv, fig. 20-24. (Lithoglyphus zonatus, de Woodward, 1859.)

35. Spekia Duveyrieriana, Bourg., Moll. Giraud Tang., p. 37, 1885, et Mal. Tang., pl. v, fig. 4-6.

36. Spekia Grandidieriana, Bourg., Loc. sup. cit., p. 40, 1885, et Mal. Tang., pl. v, fig. 7-9.

37. Spekia Cameroni, Bourg., Mal. Tang., pl. v, fig. 13-15.

38. Spekia Giraudi, Bourg., Moll. Giraud Tang., p. 36, 1885, et Mal. Tang., pl. Iv, fig. 25-27. 
39. Spekia Hamyana, Bourg., Loc. sup. cit., p. 38, 1885, et Mal. Tang., pl. v, fig. 1-3.

40. Spekia Reymondi, Giraud, in : Bourg., Loc. sup. cit., p. 39, 1885, et Mal. Tang., pl. v, fig. $10-12$.

\section{AMPULLARIDF}

41. Ampullaria Bridouxi, Bourg., Mal. Tang., pl. v, fig. 22.

42. Ampullaria ovata, Olivier, Voy. emp. ottom., II, p. 39 et atlas, pl. xxxı, fig. 1, 1804, et Bourg., Mal. Tang., pl. vi, fig. 1.

43. Meladomus Jouberti, Bourg., Mal. Tang., pl. vi, fig. 6 .

44. Meladomus sinistrorsus, Bourg., Moll. Égypte, Abyss., p. 39, 1879. (Paludina sinistrorsa, Lea, in : Trans. amer. phil. Soc. vi, et Observ. q. Unio, II, p. 90, pl. xxıI, fig. 78.)

45. Leroya Bourguignati, Grandidier, in : Bull. Soc. mal., Fr., IV, 1887, p. 192, et Bourg., Mal. Tang., pl. vi, fig. 2-5.

\section{HAUTTEGOEURID $E$}

46. Tanganikia Fagotiana, Bourg., Moll. Giraud Tang., p. 43, 1885, et Mal. Tang., pl. v, fig. 20-21.

47. Tanganikia opalina, Bourg., Mal. Tang., pl. v', 18-19.

48. Tanganikia Giraudi, Bourg., Mal. Tang., pl. v, fig. 16-17.

49. Cambieria rufofilosa, Bourg., Mal. Tang., pl. vi, 
fig. 8-10. (Lithoglyphus rufofilosus, Smith., 1880. Tanganikia rufofilosa [non Crosse], Bourg., 1885. )

50. Cambieria Maunoiriana, Bourg., Mal. Tang., pl. vi, fig. 11-12. (Tanganikia Maunoiriana, Bourg., 1885.j

51. Cambieria ovoidea, Bourg,, Mal. Tang.,pl. vi, fig. 13 -14. ( Tanganikia ovoidea, Bourg., 1885.)

52. Cambieria Jouberti, Bourg., Mal Tang., pl. vi, fig. 15-16.

53. Hauttecouria Hamyana, Bourg., Moll. Giraud Tang., p. 48, 1885, et Mal. Tang., pl. vir, fig. 6-7.

54. Hauttecœuria Burtoni, Bourg., Mal. Tang., pl. viI, fig. 1-3.

55. Hauttecœuria Moineti, Bourg., Mal. Tang., pl. vi, fig. 4-5.

56. Hauttecouria macrostoma, Bourg., Mal. Tang., pl. vi, fig. 10-11.

57. Hauttecouria Giraudi, Bourg., Moll. Giraud Tang., p. 49, 1885, et Mal. Tang., pl. viI, fig. 8-9.

58. Hauttecouria Milne-Edwardsiana, Bourg., Moll. Giraud Tang., p. 50, 1885, et Mal.Tang., pl. viI, fig. 12-14.

59. Hauttecouria Charmettanti, Bourg., Mal., Tang., pl. vir, fig. 15-17.

60. Hauttecouria soluta, Bourg., Moll. Giraud Tang., p. 51, 1885, et Mal. Tang., p,. viI, fig. 18-19. 
61. Hautlecocuria Brincatiana, Bourg., Mal. Tang., pl. vir, fig. 20-21.

62. Hauttecœuria singularis, Bourg., Moll. Giraud Tang., p. 52, 1885, et Mal. Tang., pl. viI, fig. 22-23.

63. Hauttecocuria Jouberti, Bourg., Mal. Tang., pl. vir, fig. 24-25.

64. Hauttecouria Duveyrieriana, Bourg., Moll. Giraud Tang., p. 53, 1885, et Mal. Tang., pl. viI, fig. 4-6.

65. Hautlecocuria Reymondi, Giraud, in : Bourg., Moll. Giraud Tang., p. 54, 1885, et Mal. Tang., pl. viII, fig. 1-3.

66. Hauttecouria Maunoiriana, Bourg., Moll. Giraud Tang., p. 55, 1885, et Mal. Tang., pl. virI, fig. 7-8.

67. Hauttecouria Levesquiana, Bourg., Mal. Tang., pl. viII, fig. 9-11.

68. Hauttecouria Locardiana, Bourg., Mal. Tang., pl. viri, fig. 12-14.

69. Hauttecouria Lavigieriana, Bourg., Mal. Tang., pl. viI, fig. 26-27.

70. Hauttecouria Servainiana, Bourg., Mal.Tang., pl. viII, fig. 18-19.

71. Hauttecouria Cameroni, Bourg., Mal. Tang., pl. viri, fig. 15-17.

72. Hauttecouria pusilla, Bourg., Mal. Tang., pl. viII, fig. 32-34.

73. Hauttecouria eximia, Bourg., Moll. Giraud Tang., p. 55, 1885, et Mal. Tang., pl. virl, fig. 20-22. 
74. Hauttecouria Cambieri, Giraud, in : Bourg., Moll. Giraud Tang., p. 56, 1885, et Mal. Tang., pl. viII, fig. 23-25.

75. Hauttecouria Bridouxiana, Bourg., Mal. Tang., pl. viII, fig. 26-28.

76. Hauttecouria minuta, Bourg., Moll. Giraud Tang., p. 57, 1885, et Mal. Tang., pl. viII, fig. 29-31.

\section{HYLACANTHID $\&$}

77. Hylacantha Horei, Ancey, in : Natur., 1886. (Tiphobia Horei, Smith, in: Proceed. zool. Soc. Lond., p. 348, pl. xxxı ; fig. 6-6 ${ }^{\text {B }}$, et p. 293 , pl. Lxxiv, fig. 28, 1883, et Bourg, in : Bull. Soc. mal. Fr., III, 1886, p. 143, pl. vi, fig. 1-4, et Mal. Tang., pl. Ix, fig. 1-4.)

78. Hylacantha longirostris, Bourg., in : Bull. Soc. mal. Fr., III, p. 144, pl. vi, fig. 8-10, 1886, et Mal. Tang., pl. Ix, fig. 8-10.

79. Hylacantha Jouberti, Bourg., in : Bull. Soc. mal. Fr., III, p. 146, pl. vi, fig. 11-13, 1886, et Mal. Tang., pl. Ix, fig. 11-13.

80. Hylacantha Bourguignati, Joubert, in : Bourg., in : Bull. Soc. malac. Fr., III, p. 148, pl. vi, fig. 5-7, 1886, et Mal. Tang., pl. Ix, fig. 5-7.

\section{LITTORINIDE}

81. Limnotrochus Kirki, Smith., in : Ann. and mag. nat. Hist., N. s. vi, 1880, p. 426 et in : Proc. zool. Soc. Lond., 1881, p. 286, pl. xxxıI, fig. 18-18^, et Bourg., Mal. Tang., pl. x, fig. 1-3. 
82. Limnotrochus Thomsoni, Smith., Loc. sup. cit., 1880 , p. 425 , et 1881 , p. 285 , pl. xxxıI, fig. 17-17в ; et Bourg., Mal. Tang., pl. x, fig. 4-7.

83. Limnotrochus Giraudi, Bourg., Moll. Giraud Tang., p. 59, 1885, et Mal. Tang., pl. x, fig. 8-10.

84. Limnotrochus cyclostoma, Bourg., Moll. Giraud Tang., p. 60, 1885, et Mal. Tang, pl. x, fig. 11-13.

\section{SYRNOLOPSIDE}

85. Syrnolopsis lacustris (non Crosse), Smith, in: Ann. and mag. nat. Hist., 1880, p. 426, et in : Proc. zool. Soc. Lond., 1881, p. 283, pl. xxxıI, fig. 21-21 ${ }^{\mathrm{B}}$, et Bourg., Mal. Tang., pl. x, fig. 14-17.

86. Syrnolopsis Hamyana, Bourg., Moll. Giraud Tang., p. 17, 1885, et Mal. Tang., pl. x, fig. 18-21.

87. Syrnolopsis Grandidieriana, Bourg., Moll. Giraud Tang., p. 18, 1885, et Mal. Tang., pl. x, fig. 22-24. (Syrnolopsis lacustris de Crosse, non Smith.)

88. Syrnolopsis Anceyana, Bourg., Moll. Giraud Tang., p. 20, 1885, et Mal. Tang., pl. x, fig. 25-27.

89. Syrnolopsis Giraudi, Bourg., Moll. Giraud Tang., p. 20, 1885, et Mal. Tang., pl. x, fig. 31-33.

90. Syrnolopsis minuta, Bourg., Moll. Giraud Tang. p. 21, 1885, et Mal. Tang., pl. x, fig. 28-30. 
GIRAUDIDA

91. Giraudia præclara, Bourg., Moll. Giraud Tang., p. 62, 1885, et Mal. Tang., pl. XI, 16-18.

92. Giraudia Grandidieria, Bourg., Moll. Giraud Tang., p. 63, 1885, et Mal. Tang., pl. xI, fig. 19-21.

93. Giraudia Lavigeriana, Bourg., Mal. Tang., pl. xi, fig. 22-24.

94. Reymondia Horei, Bourg., Moll. Giraud Tang., p. 65, 1885, et Mal. Tang., pl. xi, fig. 1-2 (melania? Horei de Smith, 1880).

95. Reymondia Giraudi, Bourg., loc. sup. cit., p. 65, 1885, et Mal. Tang., pl. xI, fig. 3-4.

96. Reymondia Jouberti, Bourg., Mal. Tang., pl. xI, fig. 5-6.

97. Reymondia Monceti, Bourg., Mal. Tang., pl. xI, fig. 7-8.

98. Reymondia Bridouxiana, Bourg., Mal. Tang., pl. xI, fig. 14-15.

39. Reymondia pyramidalis, Bourg., Mal. Tang., pl. xi, fig. 9-13.

\section{MELANID $F$}

100. Melania tuberculata, Bourg., Moll. orient., p. 65, 1853, et Mal. Alg., II, 1864, p. 25, pl. xv, fig. 1-11, et Mal. Tang., pl. xi, fig. 2627 (nerita tuberculata, Müller, 1774).

101. Melania admirabilis, Bourg., Mal. Tang., 
pl. xI, fig. 25. (Melania [Sermyla] admirabilis, Smith, in: Ann. and. mag. nat hist., 1880, p. 427.)

102. Horea tanganikana, Bourg., Mal. Tang., pl. xi, fig. 28-29.

103. Bourguignatia Bridouxi, Bourg., Mal. Tang., pl. xII, fig. 1-4.

104. Bourguignatia Jouberti, Bourg., Mal. Tang., pl. xII, fig. 5-7.

105. Bourguignatia imperialis, Giraud, in : Bull. Soc. mal. Fr., 1885, p. 193, pl. virr, fig. 5-7, et Bourg., Moll. Giraud Tang., p. 67, 1885, et Mal. Tang., pl. xiI, fig. 8-10.

106. Randabelia catoxia, Bourg., Mal. Tang., pl. xıII, fig. 1-2.

107. Randabelia Hamyana, Bourg., Mal. Tang., pl. xıII, fig. 3-4. (Paramelania Hamyana, Bourg., Moll. Giraud Tang., p. 71, 1885.)

108. Joubertia Baizeana, Bourg., Mal. Tang., pl. xIII, fig. 5-7. (Paramelania Baizeana, Bourg. Moll. Giraud Tang., p. 74, 1885.)

109. Joubertia spinulosa, Bourg., Mal. Tang., pl. xıII, fig. 8-10. (Paramelania spinulosa, Bourg., Moll. Giraud Tang., p. 75, 1885.)

110. Joubertia Stanleyana, Bourg., Mal. Tang., pl. xıII, fig. 11-12. (Paramelania Stanleyana, Bourg., Moll. Giraud Tang., p. 75, 1885.)

111. Lavigeria diademata, Bourg., Mal. Tang., pl. xIII, fig. 15-17.

112. Lavigeria coronata, Bourg., Mal. Tang., pl. xIII, fig. 13-14. 
113. Lavigeria grandis, Bourg., Malac. Tang., pl. xıv, fig. 1. (Melania [Paramelania] nassa, var. B. grandis, Crosse, 1881, et Paramelania grandis, Bourg., Moll. Giraud Tang., p. 69, 1885.)

114. Lavigeria callista, Bourg., Mal. Tang. pl.xıv, fig. 2.

115. Lavigeria Jouberti, Bourg., Malac. Tang., pl. xIv, fig 4.

116. Lavigeria perexemia, Bourg., Malac. Tang., pl. xIv, fig. 3 .

117. Lavigeria combsa, Bourg., Malac. Tang., pl. xiv, fig. 7.

118. Lavigeria Ruellaniana, Bourg., Mal. Tang., pl. xiv, fig. 5 .

119. Edgaria paucicostata, Bourg., Mal. Tang., pl. xıv, fig. 8-9. (Melania [Paramelania] nassa, var.g. paucicostata, Crosse, 1881; Paramelania paucicostata, Bourg., Moll. Giraud Tang., p. 69,1885 .)

120. Edgaria callopleuros, Bourg., Mal. Tang., pl. xiv, fig. 10-11. (Paramelania callopleuros, Bourg., Moll. Giraud Tang., p. 69, 1885.)

121. Edgaria Monceti, Bourg., Mal. Tang., pl. xiv, fig. 12-13.

122. Edgaria littoralis, Bourg., Malac. Tang., pl. xIv, fig. 14-16.

123. Paramelania Damoni, Smith, in : Proceed. zool. Soc. Lond., p. 559, fig. 1, 1881, et Bourg. Mal. Tang., pl. xiv, fig. 17.

124. Paramelania crassigranulata, Smith, in : 
Proceed. zool. Soc. Lond., 1881, p. 560, fig. 2, et Bourg., Mal. Tang., pl. xiv, fig. 18. 125. Paramelania infralirata, Bourg., Mal. Tang., pl. xv, fig. 4-5.

126. Paramelania egregia, Giraud, in : Bourg., Moll. Giraud Tang., p. 81, 1885, et Mal. Tang., pl. xv, fig. 1-3.

127. Paramelania obtusa, Bourg., Mal. Tang., pl. xv, fig. 6-8.

128. Paramelania Duveyrieriana, Giraud, in : Bourg., Moll. Giraud Tang., p. 79, 1885, et Mal. Tang., pl. xv, fig. 12-13.

129. Paramelania Ledoulxiana, Giraud, in : Bourg., Moll. Giraud Tang., p. 80, 1885, et Mal. Tang., pl. xv, fig. 14-15.

130. Paramelania formosa, Bourg., Mal. Tang., pl. $\mathrm{xv}$, fig. 9-11.

131. Paramelania Cameroniana, Bourg., Moll. Giraud Tang., p. 80, 1885, et Malac. Tang., pl. xv, fig. 22-23.

132. Paramelania singularis, Bourg., Mal. Tang., pl. xv, fig. 16-17.

133. Paramelania Bourguignati, Giraud, in : Bourg., Moll. Giraud Tang., p. 73, 1883, et Mal. Tang., pl. xv, fig. 18-19.

134. Paramelania Reymondi, Giraud, loc. sup. cit., p. 72, 1885, et Bourg., Mal. Tang., pl. xv, fig. 20-21.

135. Paramelania timida, Bourg., Mal. Tang., pl. xv, fig. 24-25.

136. Paramelania Milne-Edxardsiana, Bourg., 
Moll. Giraud Tang., p. 77, 1885, et Mal. Tang., pl. xv, fig. 30 .

137. Paramelania Lessepsiana, Giraud, in : Bourg., Moll. Giraud Tang., p. 78, 1885, et Mal. Tang., pl. xv, fig. 28-29.

138. Paramelania palustris, Bourg., Mal. Tang., pl. xv. fig. 31-32.

139. Paramelania Grandidieriana, Bourg., Moll. Giraud Tang., p. 70, 1885, et Mal. Tang., pl. xvi, fig. 1-2.

140. Paramelania nassatella, Bourg., Mal. Tang., pl. xvi, fig. 3-4.

141. Paramelania lacunosa, Bourg., Mal. Tang., pl. xvi, fig. 5-6.

142. Paramelania Randabeli, Bourg., Mal. Tang., pl. xvi, fig. 21-22.

143. Paramelania nassa, Bourg., Moll. Giraud Tang., p. 67, 1885, et Mal. Tang., pl. xvi, fig. 7-8. (Melania [Melanella] nassa, de Woodward, 1859.)

144. Paramelania Smithi, Bourg., Mal. Tang., pl. xvi, fig. 11-12.

145. Paramelania venusta, Bourg., Mal. Tang., pl. xvi, fig. 13-14. (Melania nassa [non Woodward] de Brot, 1874.)

146. Paramelania Mabilliana, Bourg., Mal. Tang., pl. xvi, fig. 17-18.

147. Paramelania Livingstoniana, Giraud, in : Bourg., Moll. Giraud Tang., p. 85, 1885, et Mal. Tang., pl. xvir, fig. 1-2.

148. Paramelania nassatiformis, Bourg., Mal. 
Tang. pl. xvir, fig. 5-6. (Melania [Melanella] nassa [non Woodward] de Smith, 1881.)

149. Paramelania Limnæa, Bourg., Mal. Tang., pl. xvIr, fig. 7-8.

150. Paramelania pulchella, Bourg., Moll. Giraud Tang., p. 86, 1885, et Mal. Tang., pl. xviI, fig. 9-10.

151. Paramelania elongata, Bourg., Mal. Tang., pl. xvi, fig. 9-10.

152. Paramelania Giraudi, Bourg., Moll. Giraud Tang., p. 82, 1885, et Mal. Tang., pl. xvi, fig. 19-20.

153. Paramelania Locardiana, Bourg., loc. sup. cit., p. 82, 1885, et Mal. Tang., pl. xvi, fig. 2324.

154. Paramelania crassilabris, Bourg., loc. sup. cit., p. 84, 1885, et Mal. Tang., pl. xvi, fig. 1516.

155. Paramelania bythiniformis, Bourg.., Mal. Tang., pl. xv, fig. 26-27.

156. Paramelania Servainiana, Bourg., Moll. Giraud Tang., p. 83, 1885, et Mal. Tang., pl. xvir, fig. 11-12.

157. Paramelania arenarum, Bourg., Mal. Tang., pl. xvII, fig. 3-4.

\section{NERITID E}

158. Stanleya neritoides, Bourg., Moll. Giraud Tang., p. 87, 1885, et Mal. Tang., pl. xviI, fig. 13-15. (Lithoglyphus neritoides, de Smith, 
1880 ; Tanganyicia? neritoides, de Crosse, 1881.)

159. Coulboisia Giraudi, Bourg., Mal. Tang., pl. xvir, fig. 16-17. (Stanleya Giraudi, Bourg., Moll. Giraud Tang., p. 88, 1885.)

160. Coulboisia Smithiana, Bourg., Mal. Tang., pl. xvir, fig. 18-19. (Stanleya Smithiana, Bourg., Moll. Giraud Tang., p. 88, 1885.)

\section{NATICIDE}

161. Rumella globosa, Bourg., Mal. Tang., pl. xvII, fig. 20-22.

162. Rumella callifera, Bourg., Mal. Tang., pl. xvir, fig. 23-25.

163. Rumella Milne-Edwardsiana, Bourg., Moll. Giraud Tang., p. 91, 1885, et Mal. Tang., pl. xvII, fig. 26-28.

164. Rumella Giraudi, Bourg., loc. sup. cit., p. 90, 1885, et Mal. Tang., pl. xvir, fig. 35-37.

165. Rumella Jouberti, Bourg., Mal. Tang., pl.xviI, fig. 29-31.

166. Rumella Lavigeriana, Bourg., Mal. Tang., pl. xvir, fig. 32-34.

\section{ACEPHALA}

\section{LAMELLIBRANCHIATA}

SPHERIDE

167. Grandidieria elongata, Bourg., Moll. Giraud Tang., p. 94, 1885. 
168. Grandidieria Giraudi, Bourg., Moll. Giraud Tang., p. 95, 1885.

169. Grandidieria mira, Bourg., Moll. Giraud Tang., p. 96, 1885.

170. Grandidieria Servainiana, Bourg., in : Bull. Soc. mal. Fr., II, 1885, p. 6. (Unio Burtoni [non Woodward], Smith, in : Proceed. zool. Soc. Lond., 1881, pl. xxxiv, fig. 33 seulement.)

171. Grandidieria Horei, Bourg., in : Bull. Soc. mal Fr., p. 6, 1885. (Unio Horei, Smith, in : Proceed. zool. Soc. Lond., 1881, pl. xxxiv, fig. 37.)

172. Grandidieria insignis, Ancey, in : Bourg. Esp. nouv. et g. nouv., Ouk. et Tang., p. 16, 1885.

173. Grandidieria rotundata, Bourg., Moll. Giraud Tang., p. 98, 1885.

174. Grandidieria Burtoni, Bourg., in : Bull. Soc. mal. Fr., II, 1885, p. 6. (Unio Burtoni [non Smith, non Crosse ], Woodward, in : Proceed. Soc. Lond., 1859, p. 349, pl. xuvir, fig. 1.)

175. Grandidieria Smithi, Bourg., in : Bull. Soc. mal. Fr. II, 1885, p. (Unio Burtoni [non Woodward], Smith, in : Proced. zool. Soc. Lond., 1881, pl. xxxiv, fig. $33^{\mathrm{A}}$ seulement.)

176. Grandidieria Anceyi Bourg., Esp. nouv. et g. nouv. Ouk. et Tang., p. 15, 1885.

177. Grandidieria callista, Bourg., Mal. Tang., pl. xvin, 1888.

178. Grandidieria cyrenopsis, Bourg., in : Bull. Soc. mal. Fr., p. 9., pl. I, fig. 7-9, 1885. 
179. Grandidieria Hauttecœuri Bourg., Esp. nouv. et g. nouv. Ouk. et Tang., p. 17, 1885.

180. Grandidieria singularis, Bourg., Mal. Tang., pl. xix, 1888.

181. Grandidieria Thomsoni, Bourg., in : Bull. Soc. mal. Fr. II, 1885, p. 7. (Unio Thomsoni, Smith, in : Proceed. zool. Soc. Lond., p. 399, pl. xxxıv, fig. 36, 1881.)

182. Grandidieria Ujïensis, Bourg., in : Bull. Soc. mal. Fr. II, 1885, p. 7. (Unio Nyassaensis [ non Lea], var Tanganyicensis, Smith, in : Proc. zool. Soc. Lond. 1881, pl. xxxiv, fig. $34^{\mathrm{A}}$ seulement, et [idem], var. Ujijensis de Crosse. 1881.)

183. Grandidieria corbicula, Bourg., Moll. Giraud Tang., p. 100, 1885, et Mal. Tang., pl. xix.

184. Grandidieria incarnata, Bourg., Moll. Giraud Tang., p. 101, 1885.

185. Grandidieria granulosa, Bourg, Moll. Giraud Tang., p. 102, 1885, et Mal. Tang., pl. xIx.

186. Grandidieria tanganikana, Bourg., in : Bull. Soc. mal. Fr. II, 1885, p. 7. (Unio tanganyicensis, Smith, in : Proc. zool. Soc. Lond., 1880 , p. 351, pl. xxxi, fig. 9 et $9^{\text {A }}$, et 1881 , pl. xxxiv, fig. 35.)

187. Grandidieria Locardiana, Bourg., Esp. nouv. et g. nouv. Ouk. et Tang., p. 19, 1885.

188. Grandidieria gravida, Bourg., in : Bull. Soc. mal. Fr. II, 1885, p. 7, pl. I, fig. 1-6.

189. Grandidieria rostrata, Bourg., in : Bull. Soc. mal. Fr. II, 1885, p. 10, pl. I, fig. 10-12. 
190. Corbicula tanganikana, Bourg., Moll. Giraud Tang., p. 104, 1885. (Cyrena [Corbicula] radiata, var. Smith, in : Proc. zool. Soc. Lond., 1881, p. 295, et var. Tanganyicensis de Crosse.)

191. Pisidium Giraudi, Bourg., Moll. Giraud Tang., p. 105, 1885.

192. Pisidium Hermosum, Bourg., Mal. Tang., pl. xvIII, 1888.

\section{UNIONIDA}

193. Unio calathus, Bourg., Esp. nouv. et g. nouv. Oukéréwé et Tang., p. 23, 1885.

194. Unio Jouberti, Bourg., Univ. et Irid. Tang., p. 8, 1886.

195. Unio Charbonnieri, Bourg., Univ. et Irid. Tang., p. 9, 1886, et Mal. Tang., pl. xx, fig. 1-2.

196. Unio Moineti, Bourg., Un. et Irid. Tang., p. 11, 1886.

197. Unio Coulboisi, Bourg., Un. et Irid. Tang., p. 12, 1886, et Mal. Tang., pl. xx, fig. 3-4.

198. Unio Bridouxi, Bourg., Un. et Irid. Tang., p. 13, 1886.

199. Unio Lavigerianus, Bourg., Un. et Irid., Tang., p. 14, 1886.

200. Unio Guillemeti, Bourg., Un. et Irid. Tang., p. 15, 1886.

201. Unio Dromauxi, Bourg., Un. et Irid. Tang., p. 17, 1886, et Mal. Tang., pl. xxi, fig. 1-2. 
202. Unio Vynckei, Bourg., Un. et Irid., Tang., p. 18, 1886.

203. Unio Josseti, Bourg., Un. et Irid. Tang., p. 19, 1886.

204. Unio Menardi, Bourg., Un. et Irid. Tang., p. 20, 1886.

205. Unio Vysseri, Bourg., Un. et Irid. Tang., p. 21, 1886.

206. Unio Randabeli, Bourg., Un. et Irid. Tang., p. 22, 1886.

IRIDINIDA

207. Mutela Bridouxi, Bourg., Un. et Irid. Tang., p. 25, 1886, et Mal. Tang., pl. xiI, fig. 1.

208. Mutela Lavigeriana, Bourg., Un. et Irid, Tang., p. 26, 1886.

209. Mutela soleniformis, Bourg., Esp. nouv. et g. nouv., Ouk. et Tang., p. 25, 1885, et Mal. Tang., pl. xII, fig. 2-3. (Mutela exotica? de Smith; in : Proc. zool. Soc. Lond., 1880, p. 350 , et 1881, p. 296.)

210. Mutela Moineti, Bourg., Un. et Irid. Tang., p. 27, 1886.

211. Mutela Jouberti, Bourg., Un. et Irid. Tang., p. 28, 1886, et Mal. Tang., pl. xxin, fig. 1.

212. Mutela Monceti, Bourg., Un. et Irid. Tang., p. 29, 1886.

213. Mutela Vysseri, Bourg., Un. et Irid. Tang., p. 31, 1886, et Mal. Tang., pl. xxıI, fig. 2-3. 214. Burtonia tanganikana, Bourg., Moll. Ouk. p. 20, 1883, et Esp. nouv. et g. nouv. Ouk. et 
Tang., p. 38, 1885. (Spatha tanganyicensis Smith, in: Proc. zool. soc. Lond., 1880, pl. xxxı, fig. 8 seulement).

215. Burtonia Livingstoniana, Bourg., Moll. Ouk. p. 23, 1883, et Esp.nouv. et g. nouv. Ouk. et Tang., p. 38, 1885. (Spatha tanganyicensis, Smith, in : Proc.zool. Soc. Lond., 1880, pl. xxxı, fig. $8^{\mathrm{A}}$ [ non adulte], et 1881, pl. xxxiv, fig. 32 [adulte]).

216. Burtonia Moineti, Bourg., Un. et Irid. Tang., p. 33, 1886, et Mal. Tang., pl. xxv, fig. 3.

217. Burtonia elongata, Bourg., Un. et Irid. Tang., p. 34, 1886, et Mal. Tang., pl. xxv, fig. 3.

218. Burtonia subtriangularis, Bourg. Un. et irid. Tang., p. 35, 1886, et Mal. Tang., pl. xxv, fig. 2.

219. Burtonia Lavigeriana, Bourg., Un. et Irid. Tang., p. 36, 1886, et Mal. Tang., pl. xxiv, fig. 1-4.

220. Burtonia Bridouxi, Bourg., Un. et Irid. Tang., p. 37, 1886.

221. Burtonia Bourguignati, Ancey, in Bourg., Un. et Irid Tang., p. 38, 1886, et Mal Tang., xxvi, fig. 4-5.

222. Burtonia contorta, Bourg., Un. et Irid. Tang., p. 39, 1886, et Mal. Tang., xxvi, fig. 3-5.

223. Burtonia Jouberti, Bourg., Un. et Irid. Tang. p. 40, 1886.

224. Burtonia magnifica, Bourg., Un. et Irid. Tang., p. 41, 1886, et Mal. Tang., pl. xxvi, fig.1-2.

225. Burtonia Grandidieriana, Bourg., Un. et Irid. 
Tang. p. 42, 1886, et Mal. Tang., pl. xxviI, fig. 1-3.

226. Brazzæa ventrosa, Bourg., Un. et Irid. Tang., p. 45, 1886, et Mal. Tang., pl xxix, fig. 4.

227. Brazzxa Anceyi, Bourg., Esp. nouv. et g. nouv. Onk. et Tang., p. 33, 1885, et Mal. Tang. pl. xxvin, fig 1-4.

228. Brazzæa Randabeli, Bourg., Un. et Irid. Tang., p. 46, 1886.

229. Brazzæa Moineti, Bourg., Un. et Irid. Tang., p. 47.1886.

230. Brazzæa Jouberti, Bourg., Un. et Irid. Tang., p. 48, 1886.

231. Brazzæa Coulboisi, Bourg., Un. et Irid. Tang., p. 50, 1886, et Mal. Tang., pl. xxıx, fig. 1.

232. Brazzæa elongata, Bourg., Un. et Irid. Tang. p. 51, 1886, et Mal. Tang. pl. xxix, fig. 2.

233. Brazzxa Charbonnieri, Bourg., Un. et Irid. Tang., p. 52, 1886.

234.Brazzæa Lavigeriana, Bourg., Un. et Irid. Tang., p. 53, 1886.

235. Brazzæa Bridouxi, Bourg., Un. et Irid. Tang., p. 54, 1886.

236.Brazzxa Nexcombiana, Ancey, in : Bourg., Un. et Irid. Tang., p. 55, 1886.

237. Brazzxa eximia, Bourg., Un. et Irid. Tang., p. 57, 1886, et Mal. Tang., pl. xxix, fig, 5.

238.Brazzæa Bourguignati, Joubert, in : Bourg., Un. et Irid. Tang., p. 58, 1886, et Mal. Tang., pl. xxviII. fig. 5-6. 
239. Moncetia Anceyi, Bourg., Esp. nouv. et g. nouv. Ouk. et Tang., p. 35, 1885, et Mal. Tang., pl. $\mathrm{xxx}$, fig. 1-3.

240. Moncetia Lavigeriana, Bourg., Un. et Irid. Tang., p.60, 1886, et Mal. Tang., pl. xxx, fig. 6. 241. Moncetia Moineti, Bourg., Un. et Irid Tang., p. 61, 1886, et Mal. Tang., pl. xxx, fig. 7 .

242. Moncetia Rochebrunia, Bourg., Un. et Irid. Tang., p. 62, 1886, et Mal. Tang., pl. xxx, fig. 8 .

243. Moncetia Jouberti, Bourg., Un. et Irid. Tang., p. 63, 1886, et Mal. Tang., pl. xxx. fig. 4.

244. Moncetia Bridouxi, Bourg., Un. et Irid. Tang., p. 65, 1886, et Mal. Tang. pl. xxx. fig. 5.

245. Cameronia gigantea, Bourg., Un. et Irid. Tang., p. 68, 1886, et Mal. Tang., pl. xxxv, fig. 1.

246. Cameronia admirabilis, Bourg. Un. et Irid. Tang., p. 69, 1886, et Mal. Tang., pl. xxxiv, fig. 1.

247. Cameronia Bridouxi, Bourg., Un. etIrid. Tang., p. 71, 1886.

248. Cameronia Guillemeti, Bourg., Un. et Irid. Tang., p. 72, 1886.

249. Cameronia pulchella, Bourg., Un. et Irid. Tang., p. 73, 1886.

250. Cameronia Landeaui, Bourg., Un. et Irid. Tang., p. 74, 1886, et Mal. Tang., pl. xxxi, fig. 3 .

251. Cameronia obtusa, Bourg., Un. et Irid. Tang., p. $75,1886$. 
252. Cameronia complanata, Bourg., Un. et Irid. Tang., p. 76, 1886.

253. Cameronia Bourguignati, Ancey, in: Bourg., Esp. nouv. et g. nouv. Tang., p. 28, 1885, et Mol. Tang. pl. xxxıI. fig. 1.

254. Cameronia Coulboisi, Bourg., Un. et Irid. Tang., p. 77, 1886, et Mal. Tang., pl. xxxi, fig. 1-2.

255. Cameronia Locardiana, Bourg., Un. et Irid. Tang. p. 78, 1886.

256. Cameronia Revoiliana, Bourg., Moll. Giraud Tang., p. 107, 1885, et Un. et Irid. Tang., p. 79, 1886.

257. Cameronia Vynckei, Bourg., Un. et Irid.Tang., p. 81, 1886.

258. Cameronia Josseti, Bourg., Un. et Irid. Tang., p. 82, 1886, et Mal. Tang.,pl. xxxı, fig. 3.

259. Cameronia Charbonnieri, Bourg., Un. et Irid. Tang., p. 83, 1886.

260. Cameronia Spekei, Bourg., Mol.Égypte, Abyss. etc..., p. 43, 1879, et Moll. Giraud Tang., p. 106, 1885. (Iridina Spekei, Woodward, in: Proc. zool. Soc. Lond. 1859, p. 348, pl. xLVII, fig. 2.)

261. Cameronia Giraudi, Bourg., Moll. Giraud Tang., p. 107, 1885.

262. Cameronia Dromauxi, Bourg., Un. et Irid. Tang., p. 84, 1886.

263. Cameronia Lavigeriana, Bourg., Un. et Irid. Tang. p. 85, 1886.

264. Cameronia Mabilliana, Bourg., Un. et Irid. Tang., p. 86, 1886. 
265. Cameronia Jouberti, Bourg., Un. et Irid. Tang., p. 88, 1886.

266. Cameronia Marioniana, Ancey, in : Bourg., Esp. nouv. et g. nouv. Ouk. et Tang., p. 28, 1885.

267. Cameronia Moineti, Bourg., Un. et Irid. Tang., p. 89, 1886.

268. Cameronia Anceyi, Bourg., Esp. nouv. et g. nouv. Ouk. et Tang., p. 30, 1885.

269. Cameronia Randabeli, Bourg., Un. et Irid. Tang. p. 90, 1886.

270. Cameronia paradoxa, Bourg., Un. et Irid. Tang., p. 91, 1886, et Mal. Tang., pl. xxxıI, fig. 1-2.

\section{ETHERID $Æ$}

271. Atheria tanganikana, Bourg., Mal. Tang., 1888. ( Etheria elliptica [non Lamarck] de Smith in : Proc. zool. Soc. Lond., 1880, p. 352.)

\section{EXPLICATION DES PLANCHES}

\section{Planche i.}

1-2 Micronyassia eximia,

3-4 - Smithi,

5-6 - Giraudi,

7-8 - singularis,

9-10 Nyassomelania leia,

11-12 - truncatelliformis, -

13-14 - lævigata,

Bull. Soc. Malac. France. VI. Juin 1889. - 5 


$$
-66-
$$

15-16 Nyassella Smithi, Bourguignat.

$\begin{array}{llll}17-18 & - & \text { pulchra, } & - \\ 19-20 & - & \text { episema, } & - \\ 21-22 & - & \text { acuminata, } & - \\ 23-24 & - & \text { formosa, } & - \\ 25-26 & - & \text { Tayloriana. } & -\end{array}$

\section{Planghe iI}

1-2 Nyassia callista, Bourgutgnat.

3-4 - paradoxa, -

5-6 - magnifica, -

7-8 - elegans, -

9-10 - Giraudi,

11-12 - idia,

13-14 - Nyassana, -

15-16 - thaumasta, -

17-18 Nyassella arenaria,

Bull. Soc. malac. France. VI. Juin 1889. 
Bull. Soc. malac. France.VI. 1889.
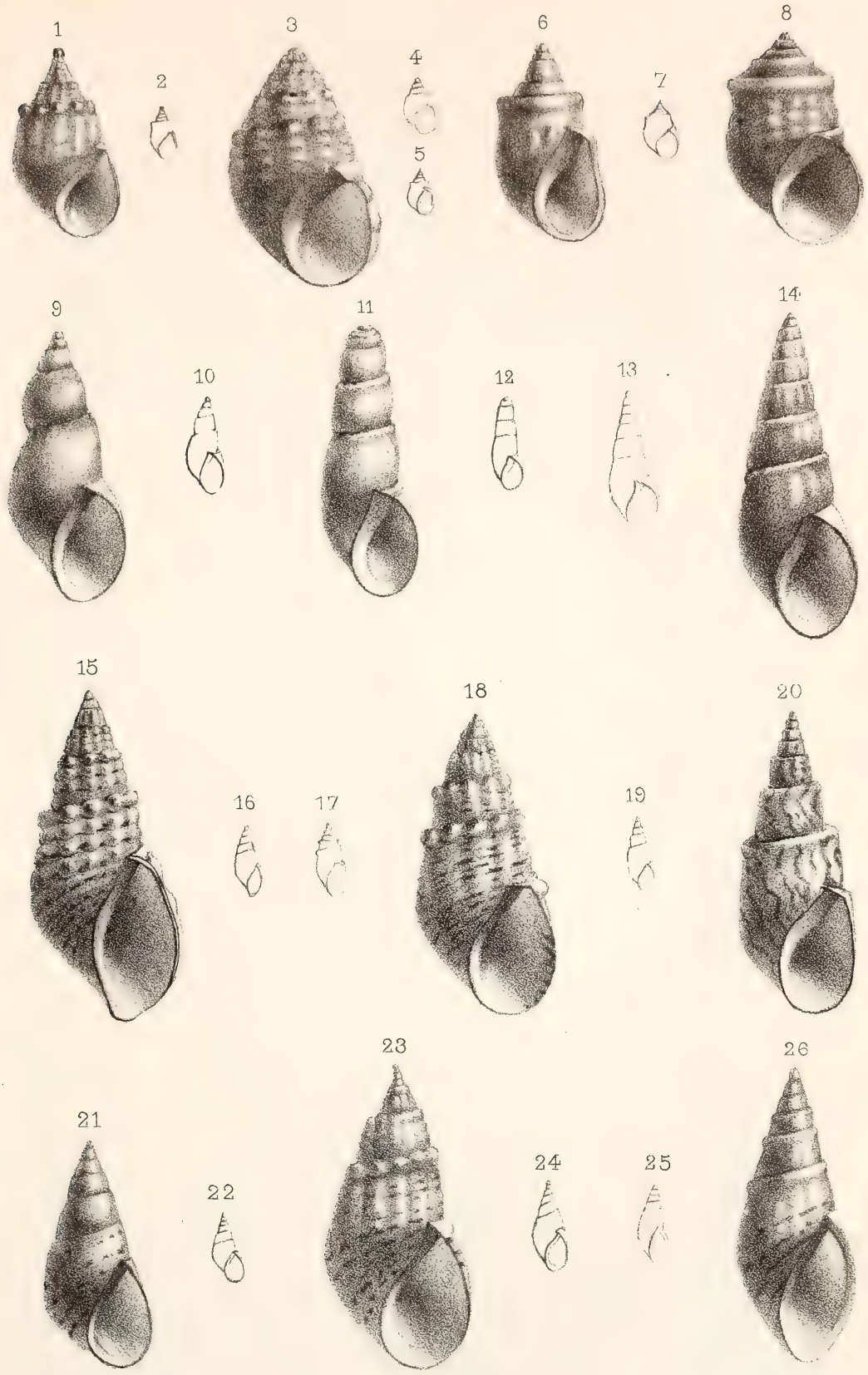

A. de Vaux-Bidon, del.

Imp. Edouard Bry, Paris.
Mélanidées
Du lac Nyassa. 

Bull. Soc. malac. France. VI. 1889
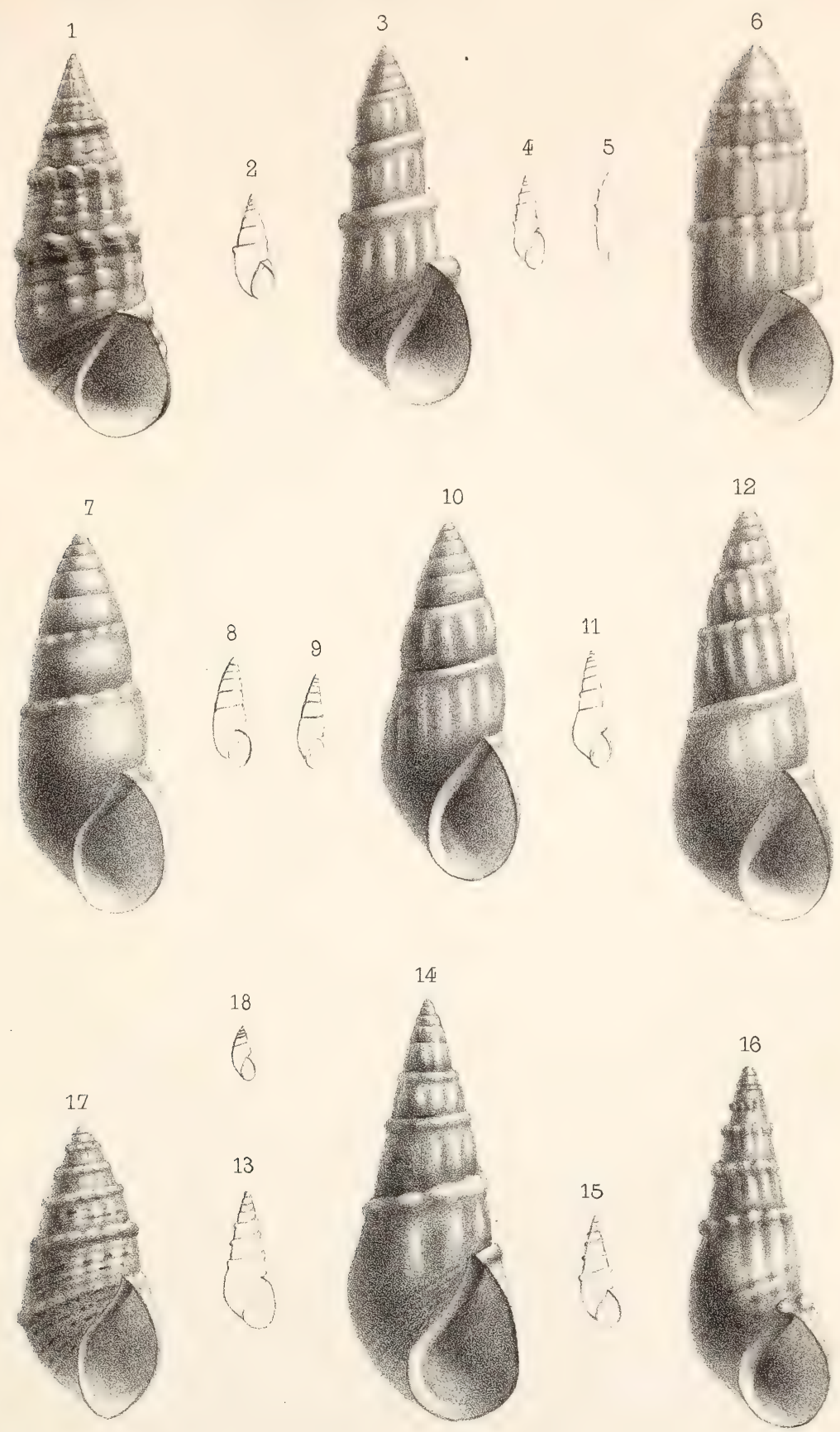

A de Vaux-Bidon, del. Imp. Edouard Bry, Paris

$$
\begin{gathered}
\text { Mélanidées } \\
\text { du lac Nyassa. }
\end{gathered}
$$







